\title{
A New Path Integral Approximation to Photon Propagation in Turbid Media
}

\author{
Rui Neves ${ }^{a, b}$ and Rui Guerra ${ }^{a}$ \\ ${ }^{a}$ Centro de Electrónica, Optoelectrónica e Telecomunicações (CEOT) \\ Faculdade de Ciências e Tecnologia, Universidade do Algarve \\ Faro, Portugal \\ Email: rguerra@ualg.pt \\ ${ }^{b}$ Unidade de Investigação Educação e Desenvolvimento (UIED) \\ Departamento de Ciências Sociais Aplicadas, Faculdade de Ciências e Tecnologia \\ Universidade Nova de Lisboa \\ Monte da Caparica, Caparica, Portugal \\ Email: rgn@fct.unl.pt
}

\begin{abstract}
The path integral approach is applied to describe the propagation of photons in bounded homogeneous turbid media. Introducing a new controlled perturbative expansion centred on the diffusion limit, new analytic solutions for continuous wave reflectance and transmittance are determined, as relevant for non-invasive measurements of tissue optical properties based on continuous wave photon migration instruments. A comparative analysis with the diffusion approximation of radiative transfer theory is made to show that non-diffusive effects are enhanced in turbid regions with increasing absorption coefficients and decreasing reduced scattering coefficients as well as when the distance between interface boundaries increases. The relation between the transport equation (which is the starting point for the path integral approach) and multiple scattering theory is also discussed.
\end{abstract}

\section{Introduction}

The analysis of photon migration in strongly scattering or turbid media has for a long time been considered in connection with the numerous fruitful applications to 
astrophysics, atmospheric optics and biomedical imaging [1]-[5]. In these studies the propagation of electromagnetic waves through a turbid medium is described by the radiative transfer theory $[1,2]$. In this theory the collective motion of the multiply scattered photons corresponds to the propagation of a radiation wave which satisfies the Boltzmann transfer equation,

$$
\left[\frac{1}{v} \frac{\partial}{\partial t}+\hat{\mathbf{s}} \cdot \nabla+\mu_{t}(\mathbf{r})\right] I(\mathbf{r}, \hat{\mathbf{s}}, t)=\mu_{s}(\mathbf{r}) \int \Psi\left(\hat{\mathbf{s}}, \hat{\mathbf{s}}^{\prime}\right) I\left(\mathbf{r}, \hat{\mathbf{s}}^{\prime}, t\right) \mathrm{d} \hat{\mathbf{s}}^{\prime}+S(\mathbf{r}, \hat{\mathbf{s}}, t),
$$

where $I(\mathbf{r}, \hat{\mathbf{s}}, t)$ is the intensity or power per unit area, per unit solid angle at point $\mathbf{r}$, from propagation direction $\hat{\mathbf{s}}$ and at time $t, S(\mathbf{r}, \hat{\mathbf{s}}, t)$ is the source function, $\mu_{s}$ is the scattering coefficient of the turbid medium and $\mu_{t}$ its transport coefficient given by $\mu_{t}=\mu_{a}+\mu_{s}$ where $\mu_{a}$ is the absorption coefficient. The photon propagation velocity $v$ is given by $v=c / n$ with $c$ the speed of light in the vaccum and $n$ the refractive index of the turbid medium. The phase function $\Psi\left(\hat{\mathbf{s}}, \hat{\mathbf{s}}^{\prime}\right)$ represents the probability density of a photon scattering from direction $\hat{\mathbf{s}}^{\prime}$ into $\hat{\mathbf{s}}$ and is normalized so that

$$
\int \Psi\left(\hat{\mathbf{s}}, \hat{\mathbf{s}}^{\prime}\right) \mathrm{d} \hat{\mathbf{s}}^{\prime}=1
$$

It should be noted that in the radiative transfer theory wave effects, such as difraction, interference and polarization, are neglected. However, the approach contains implicitly information about the correlation of fields because the intensity $I(\mathbf{r}, \hat{\mathbf{s}}, t)$ is related by a Fourier transform to the mutual coherent function defined in the analytic multiple scattering formulations beginning with the wave equations and a statistical description of the scattering media [2]. Moreover, polarization degrees of freedom may be introduced using the Stokes matrix [2]. The theory has thus a wider scope than that anticipated at first sight. Still, it remains an approximate description of the electromagnetic dynamics in turbid media with solutions based on assumptions which in many realistic situations may become too restrictive.

Appendix A provides a derivation of the transfer equation from the fundamental integral equations of multiple scattering theory. It is based on the derivation presented by Ishimaru [7] (also described in [2]). The transfer equation is obtained after three main implicit and/or explicit assumptions:

1. The scatterers are independent, in the sense that the probability of finding scatterer $s$ at $\mathbf{r}_{s}$ is independent of the position $\mathbf{r}_{t}$ of scatterer $t$.

2. The correlation function should be a slowly varying function of $\mathbf{r}$ (slow variation over the correlation distance of the field).

3. The far-field approximation should be valid for the field scattered at point $s$ when observed at any other scatterer $t$. 
This last assumption is equivalent to saying that the medium has low density on the scale of the wavelength, meaning $l \gg \lambda$, with $l$ the average inter-particle distance.

The three assumptions mentioned above are quite well satisfied by sparse particle suspensions, for example drops of water in air, polystyrene microspheres suspended in water or air bubbles trapped in a solid matrix. However, when dealing with biological media, there is a reasonable doubt that these assumptions may be sustained. Indeed, it is reasonable to keep assumption 2, but not the other two. Scattering in biological media is mainly due to the cell nucleus, the cell organelles (specially the mitochondria) and the cell walls $[8,9]$. Hence, for visible light it is $l \sim \lambda$ and assumption 3 is not valid. However, still for visible light, one may argue that condition 3 is not valid only within the volume of approximately one cell (typical size $\sim \mu \mathrm{m}$ ), containing a relatively small number of scatterers, and expect that the error introduced by still assuming condition 3 is small. Finally, the ordered structure of biological tissue means a correlated distribution of scatterers, particularly the nuclei and the cell walls. Thus, one may not sustain condition 1 (for example, the position of nucleus $t$ is not independent of position of nucleus $s$, since they are arrayed in a quasi-periodic structure). It should be noted that the correlation of the nucleus positions should be very significant for $\sim 10$ to $\sim 100$ neighbours, meaning the volume of $\sim 10^{3}$ to $\sim 100^{3}$ cells. So, in this sense, keeping assumption 1 would mean an error $\sim 10^{3}$ to $\sim 10^{6}$ times larger than the one introduced by keeping assumption 3 .

Accordingly, we tried to re-derive the transfer equation following the guidelines of [7], but introducing the effect of a pair correlation function. This is presented also in the appendix. The result is that a Boltzmann transfer equation is still obtained, but with an effective phase function obtained from the true phase function by adding a correction proportional to the Fourier transform of the pair correlation function. The interpretation of this result may be the following: the correlation between the scatterers implies that the role (on the global multiple scattering process) of radiation scattered at specific single scattering angles may be enhanced or suppressed. The effective phase function takes into account this effect. Hence, the procedures adopted in the remaining of the paper can be also applied to the case of correlated scatterers, with the adequate interpretation of the phase function term. However, the systems directly addressed in this work are suspensions of particles (not necessarily sparse) and disordered biological media such as blood or cell suspensions.

In near-infrared biomedical imaging the diffusion approximation (DA) to the Boltzmann equation [2], [6]-[15] has been extensively used. Indeed, for the propagation of near-infrared photons in biological tissue, it is not a severe reduction to consider that the mean free path of a photon is much smaller than the distance between light sources and detectors and that the absorption rate is much smaller than the scattering rate. In this conditions the number of interactions is very large and the photons may be considered to be diffusive. 
In the DA the fluence rate or power per unit area $I(\mathbf{r}, t)$ associated with the source function $S(\mathbf{r}, t)$ satisfies the following diffusion equation $[2,15]$,

$$
\frac{1}{v} \frac{\partial}{\partial t} I(\mathbf{r}, t)-\nabla \cdot[D(\mathbf{r}) \nabla I(\mathbf{r}, t)]+\mu_{a}(\mathbf{r}) I(\mathbf{r}, t)=S(\mathbf{r}, t),
$$

where $D=1 /\left[3\left(\mu_{a}+\mu_{s}^{\prime}\right)\right]$ is the diffusion coefficient with $\mu_{s}{ }^{\prime}=(1-g) \mu_{s}$ denoting the reduced scattering coefficient associated with $\mu_{s}$ and $g$, the anisotropy parameter given by

$$
g=\int \Psi\left(\hat{\mathbf{s}}, \hat{\mathbf{s}}^{\prime}\right) \hat{\mathbf{s}} \cdot \hat{\mathbf{s}}^{\prime} \mathrm{d} \hat{\mathbf{s}}^{\prime}
$$

The diffusion coefficient $D$ and the photon transport mean free path $l_{*}$ are related by $D=l_{*} / 3$.

However, random diffusive photons lose too much information to be able to produce the high quality images needed for clinical applications. In addition, realistic clinical measurements involve non-diffusive conditions such as the presence of complex sets of boundaries, the existence of turbid regions where the absorption coefficient is not negligible and the detection of photons with a short time of flight. Hence, improving the clinical relevance of near-infrared imaging requires the analysis of the Boltzmann equation beyond the DA.

One way to achieve this is to apply the Feynman path integral (PI) formalism to the analysis of the photon migration process [16]-[22]. In this approach the solution of the Boltzmann transfer equation is written as a PI involving an effective particle lagrangian $[18,19]$. The solution is constructed to preserve causality and contains information about non-diffusive photons which propagate in the continuous turbid media and scatter anisotropically. In the context of time domain systems for optical tomography, the method identified the most probable photon path around which the photon trajectories cluster and was able to generate new and accurate analytic solutions in the non-diffusive regime [18]-[22].

In this paper we extend the PI time domain analysis [18]-[22] and apply the approach to describe the migration of photons in the context of continuous wave systems built for optical tomography. In the next section we present a brief review of the PI formalism taking the opportunity to include the effect of a non-negligible absorption coefficient. In Sections 3 and 4 we introduce a new perturbative method to calculate the PI photon propagator and use it to determine new analytic solutions for the continuous wave reflectance and transmittance functions, as relevant to non-invasive measurements of tissue optical properties based on continuous wave photon migration instruments. In Section 3 we discuss the perturbative method and construct a new analytic expansion for the PI photon propagator centered on the diffusion limit. In Section 4 we apply this expansion to determine new analytic solutions for the 
continuous wave fluence rate in three representative geometric configurations: the infinite unbounded medium, the semi-infinite medium and the infinite slab geometry. The corresponding solutions for the continuous wave reflectance and transmittance are then calculated for the latter two geometries. We compare with the DA to show that non-diffusive effects are more important in turbid regions with higher absorption coefficients and lower reduced scattering coefficients. We also analyse the influence of approaching interface boundaries and show that non-diffuse effects increase when the distance between boundaries increases. In section 5 we present our conclusions.

\section{$2 \quad$ PI approach}

In the PI approach the migration of photons through a highly scattering or turbid medium is considered to be a statistical random walk problem [19]. Let us take a turbid medium characterized by an absorption coefficient $\mu_{a}$, a scattering coefficient $\mu_{s}$, an anisotropy parameter $g$ and a refractive index $n$. If the medium is homogeneous these optical parameters are simply constants and the propagation velocity of the photons in the medium is $v=c / n$ with $c$ denoting the speed of light in the vaccum.

Consider one of such photons and assume that at time $t_{i}$ it is at point $\mathbf{r}_{i}$ moving in the direction defined by the unit vector $\hat{\mathbf{s}}_{i}$. After a series of $N$ scattering events located at points $\left(\mathbf{r}_{j}, \hat{\mathbf{s}}_{j}, t_{j}\right), j=1, \cdots, N$, the photon arrives at the detection point $\left(\mathbf{r}_{f}, \hat{\mathbf{s}}_{f}, t_{f}\right)$. Suppose that at time $t_{j}$ it reaches the point $\mathbf{r}_{j}$ coming from the direction $\hat{\mathbf{s}}_{j}$ acquired after a scattering event at $\left(\mathbf{r}_{j-1}, t_{j-1}\right)$. The photon is then deflected to a direction $\hat{\mathbf{s}}_{j+1}$ by an angle $\theta_{j}=\theta\left(\hat{\mathbf{s}}_{j+1}, \hat{\mathbf{s}}_{j}\right)$ and at time $t_{j+1}$ arrives at point $\mathbf{r}_{j+1}$. Since the photon must always move with speed $v$ the probability density $P_{j}=P\left(\mathbf{r}_{j+1}, \hat{\mathbf{s}}_{j+1}, t_{j+1} ; \mathbf{r}_{j}, \hat{\mathbf{s}}_{j}, t_{j}\right)$ for this scattering event is given by

$$
P_{j}=\mathcal{N}_{j} \delta\left(\dot{\mathbf{r}}_{j}^{2}-v^{2}\right) \Psi\left(\theta_{j}\right) \exp \left(-v \mu_{a} \Delta t_{j}\right)
$$

where $\mathcal{N}_{j}$ is a normalization factor, $\delta(x)$ is the Dirac delta distribution, $\Delta t_{j}=$ $t_{j+1}-t_{j}$ and $\dot{\mathbf{r}}_{j}=\Delta \mathbf{r}_{j} / \Delta t_{j}$ with $\Delta \mathbf{r}_{j}=\mathbf{r}_{j+1}-\mathbf{r}_{j}$. The decaying exponential is the probability density for the photon not to be absorbed while moving from $\mathbf{r}_{j}$ to $\mathbf{r}_{j+1}$ when the absorption of photons is assumed to obey Fick's law. $\Psi\left(\theta_{j}\right)$ is the phase function giving the probability density for a photon with propagation direction $\hat{\mathbf{s}}_{j}$ to be deflected by an angle $\theta_{j}$ into direction $\hat{\mathbf{s}}_{j+1}$.

In an homogeneous turbid medium with optical parameters $\mu_{a}, \mu_{s}$ and $g$, the average distance travelled by a photon between each collision is the photon transport mean free path $l_{*}=1 /\left(\mu_{a}+\mu_{s}{ }^{\prime}\right)$, where $\mu_{s}{ }^{\prime}=\mu_{s}(1-g)$ is the reduced scattering coefficient. 
A small scattering angle is then approximately given by $\theta_{j} \approx\left|\Delta \mathbf{r}_{j}-\Delta \mathbf{r}_{j-1}\right| / l_{*}$. In this context the phase density is well described by a gaussian function. Then writting $\ddot{\mathbf{r}}_{j}=\left(\Delta \mathbf{r}_{j}-\Delta \mathbf{r}_{j-1}\right) / \Delta t_{j}^{2}$ and using $v \Delta t_{j} \approx l_{*}$ the probability density (5) is well approximated by

$$
P_{j}=\mathcal{N}_{j} \delta\left(\dot{\mathbf{r}}_{j}^{2}-v^{2}\right) \exp \left[-\left(v \mu_{a}+\frac{l_{*}}{2 v^{3}} \ddot{\mathbf{r}}_{j}^{2}\right) \Delta t_{j}\right]
$$

The probability density for the photon to start at point $\left(\mathbf{r}_{i}, \hat{\mathbf{s}}_{i}, t_{i}\right)$ and end at point $\left(\mathbf{r}_{f}, \hat{\mathbf{s}}_{f}, t_{f}\right)$ after the $N$ scattering events distributed by the points $\left(\mathbf{r}_{j}, \hat{\mathbf{s}}_{j}, t_{j}\right), j=$ $1, \cdots, N$, is given by the successive product of the probability densities (6) and the probability density $P_{0}=P\left(\mathbf{r}_{1}, \hat{\mathbf{s}}_{1}, t_{1} ; \mathbf{r}_{0}, \hat{\mathbf{s}}_{0}, t_{0}\right)$ associated to the unscattered propagation from $\left(\mathbf{r}_{0}, \hat{\mathbf{s}}_{0}, t_{0}\right) \equiv\left(\mathbf{r}_{i}, \hat{\mathbf{s}}_{i}, t_{i}\right)$ to $\left(\mathbf{r}_{1}, \hat{\mathbf{s}}_{1}, t_{1}\right)$,

$$
P_{0}=\mathcal{N}_{0} \delta\left(\dot{\mathbf{r}}_{0}^{2}-v^{2}\right) \exp \left(-v \mu_{a} \Delta t_{0}\right) .
$$

The probability amplitude $P_{f i}=P\left(\mathbf{r}_{f}, \hat{\mathbf{s}}_{f}, t_{f} ; \mathbf{r}_{i}, \hat{\mathbf{s}}_{i}, t_{i}\right)$ for the photon to be emitted at $\left(\mathbf{r}_{i}, \hat{\mathbf{s}}_{i}, t_{i}\right)$ and detected at $\left(\mathbf{r}_{f}, \hat{\mathbf{s}}_{f}, t_{f}\right)$ is then obtained integrating over all possible independent intermediate coordinates $\mathbf{r}_{j}, j=1, \cdots, N$. We find

$$
P_{f i}=\mathcal{N}_{f i} \int \prod_{j=1}^{N} d \mathbf{r}_{j}\left(\frac{v}{l_{*}}\right)^{N+1} \prod_{j=0}^{N} \delta\left(\dot{\mathbf{r}}_{j}^{2}-v^{2}\right) \exp \left[-\sum_{j=0}^{N}\left(v \mu_{a}+\frac{l_{*}}{2 v^{3}} \ddot{\mathbf{r}}_{j}^{2}\right) \Delta t_{j}\right],
$$

where $\mathcal{N}_{f i}$ is the probability normalization factor.

Using the exponential representation of the Dirac delta distribution,

$$
\delta\left(\dot{\mathbf{r}}_{j}^{2}-v^{2}\right)=\frac{1}{2 \pi} \int d p_{j} \exp \left[i p_{j}\left(\dot{\mathbf{r}}_{j}^{2}-v^{2}\right)\right],
$$

we obtain

$$
\begin{aligned}
P_{f i}=\mathcal{N}_{f i} \int \prod_{j=1}^{N} d \mathbf{r}_{j}\left(\frac{v}{2 \pi l_{*}}\right)^{N+1} \prod_{j=0}^{N} d p_{j} \exp \left[-\sum_{j=0}^{N}\left(v \mu_{a}+\frac{l_{*}}{2 v^{3}} \ddot{\mathbf{r}}_{j}^{2}\right) \Delta t_{j}\right] \\
\times \exp \left[i \frac{v}{l_{*}} \sum_{j=0}^{N} p_{j}\left(\dot{\mathbf{r}}_{j}^{2}-v^{2}\right) \Delta t_{j}\right] .
\end{aligned}
$$

Taking the double limit $N \rightarrow \infty, \Delta t_{j} \rightarrow 0$ we are lead to the Feynman prescription [23] for the following PI

$$
P_{f i}=\int \mathcal{D} \mathbf{r}(t) J[\dot{\mathbf{r}}(t)] \exp \left\{-\int_{t_{i}}^{t_{f}} d t\left[v \mu_{a}+\frac{l_{*}}{2 v^{3}}[\ddot{\mathbf{r}}(t)]^{2}\right]\right\}
$$


where the path density functional $J[\dot{\mathbf{r}}(t)]$ is given by

$$
J[\dot{\mathbf{r}}(t)]=\int \mathcal{D} p(t) \exp \left\{i \frac{v}{l_{*}} \int_{t_{i}}^{t_{f}} d t p(t)\left([\dot{\mathbf{r}}(t)]^{2}-v^{2}\right)\right\}
$$

and ensures that the photon velocity is kept equal to the speed of light $v$ at every point along the trajectory. The integrals over all possible intermediate points of the trajectory are constrained by the geometric configuration of the turbid region.

The PI probability given by (11) and (12) defines the photon propagator $P\left(\mathbf{r}, t ; \mathbf{r}^{\prime}, t^{\prime}\right)$ associated with the electromagnetic migration process. The fluence rate $I\left(\mathbf{r}, t ; \mathbf{r}^{\prime}, t^{\prime}\right)$ generated by a source function $S\left(\mathbf{r}, t ; \mathbf{r}^{\prime}, t^{\prime}\right)$ is then given by Huygen's principle as the time-ordered convolution of the propagator with the source,

$$
I\left(\mathbf{r}, t ; \mathbf{r}^{\prime}, t^{\prime}\right)=\int P\left(\mathbf{r}, t ; \mathbf{r}^{\prime \prime}, t^{\prime \prime}\right) S\left(\mathbf{r}^{\prime \prime}, t^{\prime \prime} ; \mathbf{r}^{\prime}, t^{\prime}\right) d \mathbf{r}^{\prime \prime} d t^{\prime \prime}
$$

\section{PI propagator}

For any geometry without a dense set of boundaries the convolution (13) is only sensitive to the propagator in the interior of the turbid region. This propagator coincides with the time domain fluence rate in an infinite medium. Indeed, in the time domain the photon source is $S\left(\mathbf{r}, t ; \mathbf{r}^{\prime}, t^{\prime}\right)=\delta\left(\mathbf{r}-\mathbf{r}^{\prime}\right) \delta\left(t-t^{\prime}\right)$ and so (13) implies that $I_{f i}=I\left(\mathbf{r}_{f}, t_{f} ; \mathbf{r}_{i}, t_{i}\right)=P_{f i}$.

Using the exact infinite series which defines the exponential function, the PI propagator (11) may be written as follows

$$
P_{f i}=\int \mathcal{D} \mathbf{r}(t) J[\dot{\mathbf{r}}(t)] \exp \left(-\int_{t_{i}}^{t_{f}} d t v \mu_{a}\right) \sum_{j=0}^{+\infty} \frac{1}{j !}\left[-\frac{l_{*}}{2 v^{3}} \int_{t_{i}}^{t_{f}}[\ddot{\mathbf{r}}(t)]^{2}\right]^{j}
$$

Close to the diffusion limit we may neglect the angular dependence of the scattering phase function. In this near diffusive regime we just keep the lowest order contribution $j=0$. Then (14) is approximated by

$$
P_{f i}=\int \mathcal{D} \mathbf{r}(t) J[\dot{\mathbf{r}}(t)] \exp \left(-\int_{t_{i}}^{t_{f}} d t v \mu_{a}\right) .
$$

The path density $J[\dot{\mathbf{r}}(t)]$ is itself a non-trivial PI. A good approximation in the isotropic regime is to consider that only the average photon speed is kept equal to $v$ and write

$$
J[\dot{\mathbf{r}}(t)]=\mathcal{N}_{J} \delta\left[\int_{t_{i}}^{t_{f}} d t\left([\dot{\mathbf{r}}(t)]^{2}-v^{2}\right)\right]
$$


where $\mathcal{N}_{J}$ is the path density normalization factor. Expanding $\mathbf{r}$ as a Fourier sine series with fundamental period $\Delta t_{f i}[23]$ the isotropic PI propagator (15) may then be shown to be given by [19]

$$
\begin{aligned}
P_{f i}= & \frac{1}{2 \pi v^{2} \Delta t_{f i}^{3}} B^{-1}\left(\frac{3}{2}, \frac{v \Delta t_{f i}}{4 D}+1\right) \exp \left(-v \mu_{a} \Delta t_{f i}\right) \\
& \times\left[1-\frac{\Delta \mathbf{r}_{f i}^{2}}{v^{2} \Delta t_{f i}^{2}}\right]^{\frac{v \Delta t_{f i}}{4 D}} \theta\left(\frac{v \Delta t_{f i}}{\left|\Delta \mathbf{r}_{f i}\right|}-1\right),
\end{aligned}
$$

where $D=l_{*} / 3$ is the diffusion coefficient, $\theta(x)$ is the unit step function and $B(\alpha, \beta)$ is the Beta function $[24,25]$. Since $P_{f i}=0$ for $\left|\Delta \mathbf{r}_{f i}\right|>v \Delta t_{f i}$ causality is automatically ensured. In (17) the normalization has been fixed by imposing the condition that scattered photons which are not absorbed must be found somewhere in space,

$$
\frac{1}{v} \int_{-\infty}^{+\infty} P_{f i} \exp \left(v \mu_{a} \Delta t_{f i}\right) d \mathbf{r}_{f}=1 .
$$

The propagator density (17) is a new PI solution to describe isotropic photon migration in turbid media with a non-zero absorption coefficient $\mu_{a}$. It naturally generalizes the corresponding propagator associated with purely scattering turbid media [19]. In figures 1 and 2 we present some examples of propagator densities $P=P(\mathbf{r}, t ; \mathbf{0}, 0)=P(r, t)$ for $r=|\mathbf{r}|=100 \mathrm{~mm}$ and $v=0.225 \mathrm{~mm} / \mathrm{ps}$.

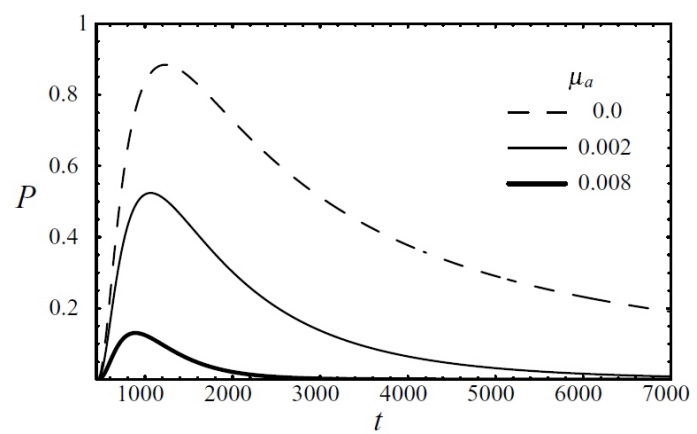

Figure 1: Plots of $P=P(100, t)$ for $\mu_{s}^{\prime}=0.05 \mathrm{~mm}^{-1} . \quad P$ is measured in units of $2.0 \times 10^{-8} \mathrm{~mm}^{-2} \mathrm{ps}^{-1}, \mu_{a}$ in $\mathrm{mm}^{-1}$ and $t$ in ps.

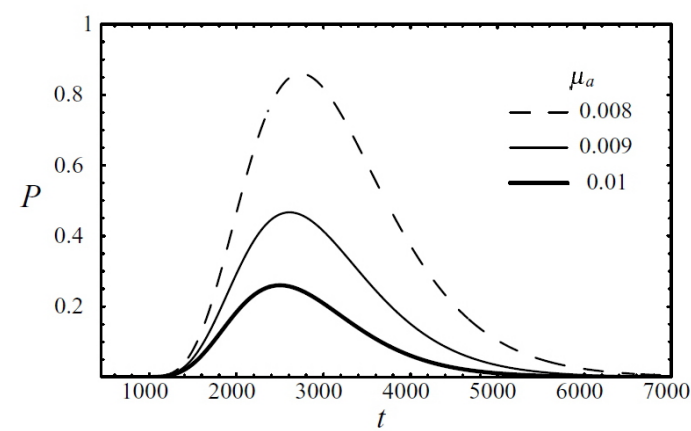

Figure 2: Plots of $P=P(100, t)$ for $\mu_{s}^{\prime}=0.5 \mathrm{~mm}^{-1}$. P is measured in units of $1.0 \times 10^{-11} \mathrm{~mm}^{-2} \mathrm{ps}^{-1}, \mu_{a}$ in $\mathrm{mm}^{-1}$ and $t$ in ps.

For fixed $\mu_{s}^{\prime}$ the propagator has a maximum intensity peak which decreases and moves towards smaller values of $t$ when $\mu_{a}$ increases. The spread around the peak 
increases with decreasing $\mu_{a}$. When $\mu_{a}$ decreases the purely scattering regime with $\mu_{a}=0$ is approached continuously. For fixed $\mu_{a}$ increasing $\mu_{s}^{\prime}$ decreases the peak intensity (note that as indicated in the plot labels the scale for $P$ is different in figures 1 and 2) and moves it towards larger values of $t$. The corresponding spread also increases. In figures 3 and 4 we consider different values of $r$ for $\mu_{s}^{\prime}=0.05 \mathrm{~mm}^{-1}$. As expected when $r$ decreases the maximum peak increases (note again the different scales for $P$ ) and is shifted to smaller values of $t$. The corresponding spread increases with $r$.

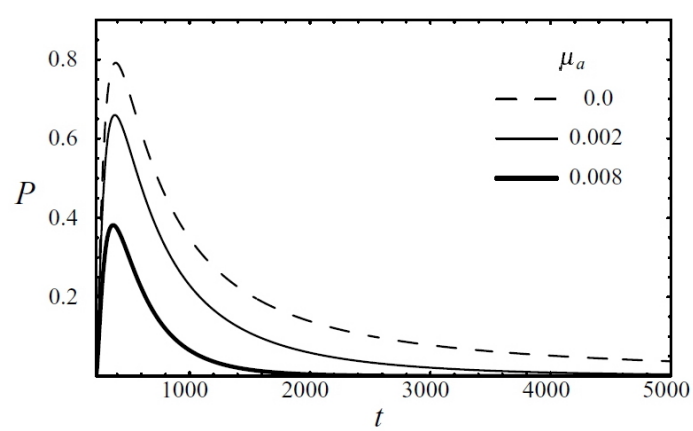

Figure 3: Plots of $P=P(50, t)$ for $\mu_{s}^{\prime}=0.05 \mathrm{~mm}^{-1} . \quad P$ is measured in units of $2.0 \times 10^{-7} \mathrm{~mm}^{-2} \mathrm{ps}^{-1}, \mu_{a}$ in $\mathrm{mm}^{-1}$ and $t$ in ps.

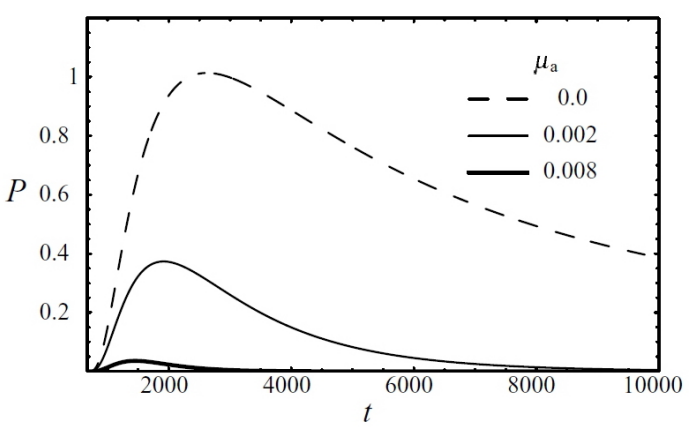

Figure 4: Plots of $P=P(150, t)$ for $\mu_{s}^{\prime}=0.05 \mathrm{~mm}^{-1} . \quad P$ is measured in units of $5.0 \times 10^{-9} \mathrm{~mm}^{-2} \mathrm{ps}^{-1}, \mu_{a}$ in $\mathrm{mm}^{-1}$ and $t$ in ps.

It should be noted that the isotropic regime of the PI approach does not reduce to the DA. This only happens in the diffusion limit around which the PI photon propagator (17) has a well defined perturbative expansion. Indeed, for late photons satisfying $\left|\Delta \mathbf{r}_{f i}\right|<<v \Delta t_{f i}$ we may write

$$
\begin{gathered}
{\left[1-\frac{\Delta \mathbf{r}_{f i}^{2}}{v^{2} \Delta t_{f i}^{2}}\right]^{\frac{v \Delta t_{f i}}{4 D}}=\exp \left[\frac{v \Delta t_{f i}}{4 D} \ln \left(1-\frac{\Delta \mathbf{r}_{f i}^{2}}{v^{2} \Delta t_{f i}^{2}}\right)\right]} \\
=\exp \left(-\frac{\Delta \mathbf{r}_{f i}^{2}}{4 v D \Delta t_{f i}}\right) \exp \left\{-\frac{\Delta \mathbf{r}_{f i}^{4}}{8 D v^{3} \Delta t_{f i}^{3}}\left[1+\frac{2 \Delta \mathbf{r}_{f i}^{2}}{3 v^{2} \Delta t_{f i}^{2}}+\mathcal{O}\left(\frac{\Delta \mathbf{r}_{f i}^{4}}{v^{4} \Delta t_{f i}^{4}}\right)\right]\right\} .
\end{gathered}
$$

On the other hand using the properties of the Gamma function [24, 25],

$$
\Gamma(z+1)=z \Gamma(z), \quad 2^{2 z-1} \Gamma(z) \Gamma\left(z+\frac{1}{2}\right)=\sqrt{\pi},
$$


we find that the Beta function satisfies

$$
B^{-1}\left(\frac{3}{2}, z+1\right)=\frac{4\left(z+\frac{3}{2}\right)\left(z+\frac{1}{2}\right) \Gamma(2 z)}{z 2^{2 z} \Gamma^{2}(z)} .
$$

When $\left|\Delta \mathbf{r}_{f i}\right|<<v \Delta t_{f i}$ we should also take $D<<v \Delta t_{f i}$. Then consider the asymptotic expansion $[24,25]$

$$
\ln \Gamma(z)=\left(z-\frac{1}{2}\right) \ln z-z+\ln \sqrt{2 \pi}+\sum_{j=1}^{n-1} \frac{B_{2 j}}{2 j(2 j-1)} z^{1-2 j}+R_{n}(z),
$$

where $n \geq 2, B_{2 j}$ are the Bernoulli numbers, $B_{2}=1 / 6, B_{4}=-1 / 30, \cdots$ and $R_{n}(z)$ is the expansion residue. Since $z=v \Delta t_{f i} /(4 D)$ is a real number the expansion residue $R_{n}(z)$ satisfies $\left|R_{n}(z)\right|<\left|B_{2 n}\right| /\left(2 n(2 n-1)|z|^{2 n-1}\right)$ and is always smaller than the last term retained in the series. To order $n=3$ we find

$$
\Gamma(z)=\exp \left[\left(z-\frac{1}{2}\right) \ln z-z+\ln \sqrt{2 \pi}\right]\left[1+\frac{1}{12 z}+\frac{1}{288 z^{2}}-\frac{139}{51840 z^{3}}+\mathcal{O}\left(\frac{1}{z^{4}}\right)\right] .
$$

Then we obtain

$$
\begin{gathered}
\Delta t_{f i}^{-3} B^{-1}\left(\frac{3}{2}, \frac{v \Delta t_{f i}}{4 D}+1\right)=2 \pi v^{3}\left(4 \pi v D \Delta t_{f i}\right)^{-\frac{3}{2}} \\
\times\left[1+\frac{15 D}{2 v \Delta t_{f i}}+\frac{65 D^{2}}{8 v^{2} \Delta t_{f i}^{2}}+\mathcal{O}\left(\frac{D^{3}}{v^{3} \Delta t_{f i}^{3}}\right)\right] .
\end{gathered}
$$

Since for $\left|\Delta \mathbf{r}_{f i}\right|<<v \Delta t_{f i}$ the step function is just equal to one (17) may be written as

$$
\begin{aligned}
& P_{f i}=P_{f i}^{\mathrm{DA}}\left[1+\frac{15 D}{2 v \Delta t_{f i}}+\frac{65 D^{2}}{8 v^{2} \Delta t_{f i}^{2}}+\mathcal{O}\left(\frac{D^{3}}{v^{3} \Delta t_{f i}^{3}}\right)\right] \\
& \times \exp \left\{-\frac{\Delta \mathbf{r}_{f i}^{4}}{8 D v^{3} \Delta t_{f i}^{3}}\left[1+\frac{2 \Delta \mathbf{r}_{f i}^{2}}{3 v^{2} \Delta t_{f i}^{2}}+\mathcal{O}\left(\frac{\Delta \mathbf{r}_{f i}^{4}}{v^{4} \Delta t_{f i}^{4}}\right)\right]\right\},
\end{aligned}
$$

where $P_{f i}^{\mathrm{DA}}=P^{\mathrm{DA}}\left(\mathbf{r}_{f}, t_{f} ; \mathbf{r}_{i}, t_{i}\right)$ is the well known solution of the time domain Boltzmann transport equation in the DA [6],

$$
P_{f i}^{\mathrm{DA}}=v\left(4 \pi v D \Delta t_{f i}\right)^{-\frac{3}{2}} \exp \left(-\frac{\Delta \mathbf{r}_{f i}^{2}}{4 v D \Delta t_{f i}}-v \mu_{a} \Delta t_{f i}\right) .
$$

In figures 5 to 8 we consider $P=P(\mathbf{r}, t ; \mathbf{0}, 0)=P(r, t)$ and compare the PI propagator (17) with its approximated expansion (API) given by (25) and the lowest order DA (26). 


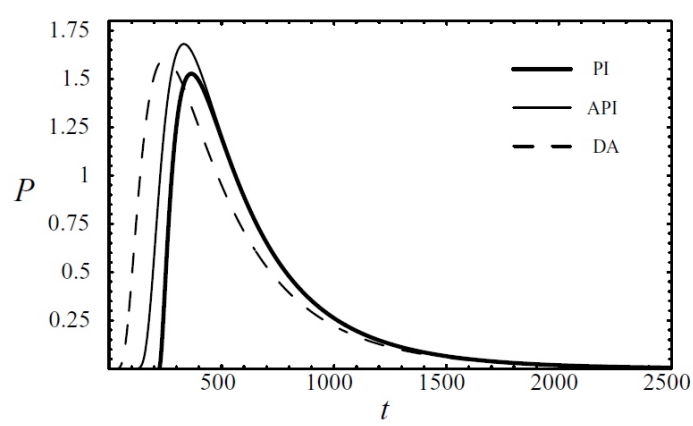

Figure 5: Plots of $P=P(50, t)$ for $\mu_{s}{ }^{\prime}=0.05 \mathrm{~mm}^{-1}$ and $\mu_{a}=0.008 \mathrm{~mm}^{-1}$. $P$ is measured in units of $5.0 \times$ $10^{-8} \mathrm{~mm}^{-2} \mathrm{ps}^{-1}$ and $t$ in ps.

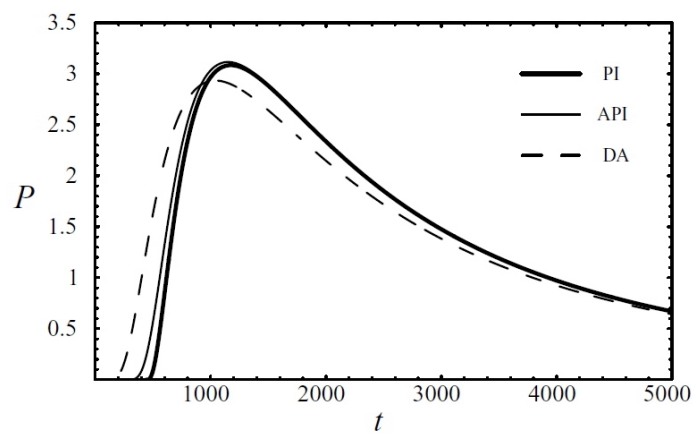

Figure 7: Plots of $P=P(100, t)$ for $\mu_{s}^{\prime}=0.05 \mathrm{~mm}^{-1}$ and $\mu_{a}=5.0 \times$ $10^{-4} \mathrm{~mm}^{-1}$. $P$ is measured in units of $5.0 \times 10^{-9} \mathrm{~mm}^{-2} \mathrm{ps}^{-1}$ and $t$ in ps.

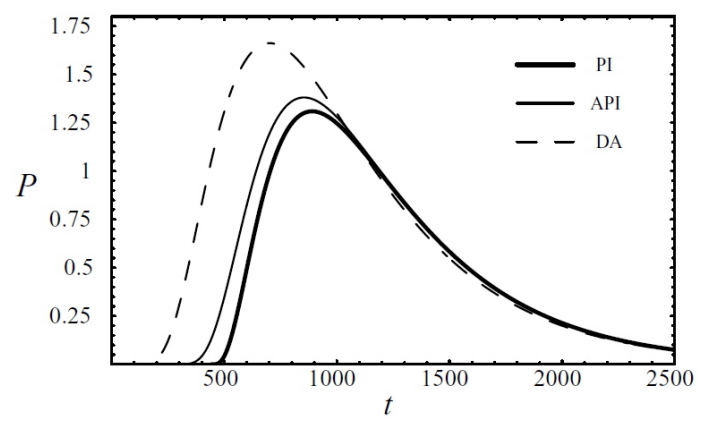

Figure 6: Plots of $P=P(100, t)$ for $\mu_{s}^{\prime}=0.05 \mathrm{~mm}^{-1}$ and $\mu_{a}=0.008 \mathrm{~mm}^{-1}$. $P$ is measured in units of $2.0 \times$ $10^{-9} \mathrm{~mm}^{-2} \mathrm{ps}^{-1}$ and $t$ in ps.

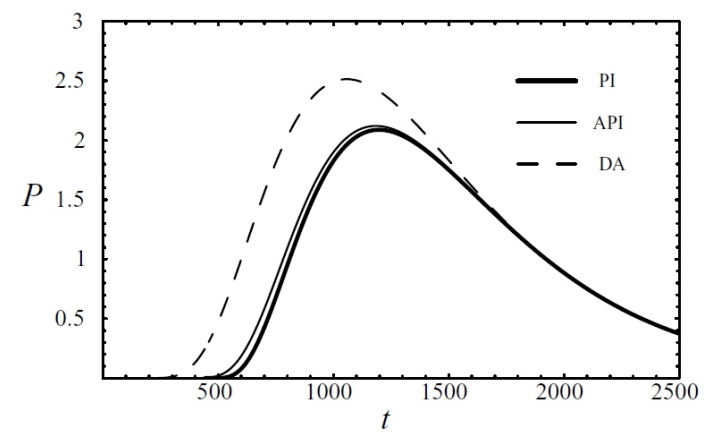

Figure 8: Plots of $P=P(100, t)$ for $\mu_{s}^{\prime}=0.1 \mathrm{~mm}^{-1}$ and $\mu_{a}=0.008 \mathrm{~mm}^{-1}$. $P$ is measured in units of $5.0 \times$ $10^{-10} \mathrm{~mm}^{-2} \mathrm{ps}^{-1}$ and $t$ in $\mathrm{ps}$.

For fixed $\mu_{s}^{\prime}$ and $\mu_{a}$ figures 5 and 6 show that when $r$ increases (25) improves as an approximation to (17). The same happens if $\mu_{a}$ decreases for fixed $r$ and $\mu_{s}^{\prime}$ (see figures 6 and 7), and if $\mu_{s}^{\prime}$ increases for fixed $r$ and $\mu_{a}$ (see figures 6 and 8 ). Figures 5 to 8 also show that (25) is clearly a better approximation than (26). Keeping enough higher order terms (25) may approximate (17) to the desired degree of accuracy. Naturally, the specific number of terms to be kept in the series depends on the turbid medium. Note that when compared to the DA the PI approach as been shown to provide a much better fit to time domain experimental data [22], 
a fact clearly establishing it as a more accurate description of photon propagation in turbid media. As a consequence, the API expansion (25) is indeed an improved approximated description of photon propagation in turbid media.

\section{Continuous wave systems}

In continuous wave systems built for optical tomography the photon source may be considered to be a Dirac delta distribution in space with constant amplitude $S$ [5]. For simplicity, let us consider that it is located at the origin $\mathbf{r}=(0,0,0)$ and that emission starts at time $t=0$,

$$
S(\mathbf{r}, t)=S \delta(\mathbf{r}) \theta(t)
$$

Because the continuous wave fluence rate depends on the geometric configuration of the turbid region let us now analyse three representative examples: the infinite medium, the semi-infinite medium and the infinite slab geometry.

\subsection{Infinite medium}

Since the photon propagator (17) is only nonzero for $\left|\Delta \mathbf{r}_{f i}\right|=r<v \Delta t_{f i}=v t$ the continuous wave fluence rate is given by

$$
I(r, t)=\frac{S}{2 \pi v^{2}} \int_{0}^{t-r / v} d \tau \frac{(t-\tau)^{-3}}{B\left[\frac{3}{2}, \frac{v(t-\tau)}{4 D}+1\right]}\left[1-\frac{r^{2}}{v^{2}(t-\tau)^{2}}\right]^{\frac{v(t-\tau)}{4 D}} \exp \left[-v \mu_{a}(t-\tau)\right],
$$

As it is the fluence rate integral is still a complicated formula which may only be determined numerically. An approximate analytic evaluation is nevertheless possible if the photon propagator is expanded around the DA. As we have seen such an expansion exists and is given by (25). With enought terms kept in the series we have shown that it is an excellent approximation to the path integral solution (17). Consequently, an accurate analytic evaluation of $I(r)$ is expected to be possible with (25). Changing the integration variable in (28) first to $y=t-\tau$ and then to $x=4 v D y / r^{2}$ we find

$$
\begin{aligned}
I(r, t) & =\frac{S}{4 D r \pi^{3 / 2}} \int_{4 D / r}^{4 D v t / r^{2}} d x x^{-3 / 2}\left[1+\frac{30 D^{2}}{r^{2} x}+\frac{130 D^{4}}{r^{4} x^{2}}+\mathcal{O}\left(\frac{D^{6}}{r^{6} x^{3}}\right)\right] \\
& \times \exp \left\{-\frac{1}{x}-\frac{\mu_{a} r^{2} x}{4 D}-\frac{8 D^{2}}{r^{2} x^{3}}\left[1+\frac{32 D^{2}}{3 r^{2} x^{2}}+\mathcal{O}\left(\frac{D^{4}}{r^{4} x^{4}}\right)\right]\right\} .
\end{aligned}
$$


Now for $D<<r$ and $v t>>r^{2} / D$ the fluence rate becomes independent of time and may be written as follows

$$
\begin{aligned}
I(r) & =\frac{S}{4 D r \pi^{3 / 2}} \int_{0}^{+\infty} d x x^{-3 / 2}\left[1+\frac{30 D^{2}}{r^{2} x}+\frac{130 D^{4}}{r^{4} x^{2}}+\mathcal{O}\left(\frac{D^{6}}{r^{6} x^{3}}\right)\right] \\
& \times \exp \left\{-\frac{1}{x}-\frac{\mu_{a} r^{2} x}{4 D}-\frac{8 D^{2}}{r^{2} x^{3}}\left[1+\frac{32 D^{2}}{3 r^{2} x^{2}}+\mathcal{O}\left(\frac{D^{4}}{r^{4} x^{4}}\right)\right]\right\} .
\end{aligned}
$$

Expanding the exponential around the diffusion limit,

$$
\begin{gathered}
\exp \left\{-\frac{1}{x}-\frac{\mu_{a} r^{2} x}{4 D}-\frac{8 D^{2}}{r^{2} x^{3}}\left[1+\frac{32 D^{2}}{3 r^{2} x^{2}}+\mathcal{O}\left(\frac{D^{4}}{r^{4} x^{4}}\right)\right]\right\}=\exp \left(-\frac{1}{x}-\frac{\mu_{a} r^{2} x}{4 D}\right) \\
\times \sum_{j=0}^{+\infty} \frac{(-8)^{j}}{j !} \frac{D^{2 j}}{r^{2 j} x^{3 j}}\left[1+\frac{32 D^{2}}{3 r^{2} x^{2}}+\mathcal{O}\left(\frac{D^{4}}{r^{4} x^{4}}\right)\right]^{j},
\end{gathered}
$$

we obtain

$$
I(r)=\frac{S}{4 D r \pi^{3 / 2}} \sum_{j=0}^{+\infty} a_{j}(r) I_{j}(r),
$$

where $I_{j}(r)$ is given in terms of the modified Bessel function of the third kind $K_{j+1 / 2}(z)[24,25]$ by

$$
\begin{aligned}
I_{j}(r) & =\int_{0}^{+\infty} d x x^{-3 / 2-j} \exp \left(-\frac{1}{x}-\frac{\mu_{a} r^{2} x}{4 D}\right) \\
& =2\left(\frac{\mu_{a} r^{2}}{4 D}\right)^{(1+2 j) / 4} K_{j+1 / 2}\left(r \sqrt{\frac{\mu_{a}}{D}}\right)
\end{aligned}
$$

and $a_{0}(r)=1, a_{1}(r)=30 D^{2} / r^{2}, a_{2}(r)=130 D^{4} / r^{4}, \cdots$.

To estimate the form of the fluence rate let us consider that besides $D<<r$ we still have $D \mu_{a}<<1$. Then expanding in the small parameter $\varepsilon \approx \mu_{a} D \approx D / r<<1$ we find

$$
I(r)=I^{\mathrm{DA}}(r)\left[1-r \sqrt{D} \mu_{a}^{3 / 2}-6 D \mu_{a}+\frac{1}{2} r^{2} D \mu_{a}^{3}+\mathcal{O}\left(\varepsilon^{3 / 2}\right)\right],
$$

where

$$
I^{\mathrm{DA}}(r)=\frac{S}{4 \pi D r} \exp \left(-r \sqrt{\frac{\mu_{a}}{D}}\right)
$$

is the DA contribution. In figures 9 and 10 we present comparative results for $\ln [r I(r)]$ taking $S=1000 \mathrm{ps}^{-1}$. Clearly, smaller reduced scattering coefficients $\mu_{s}^{\prime}$ and higher absorption coefficients $\mu_{a}$ lead to larger differences between the DA and 
API. Note that both $\mu_{a}$ and $1 / \mu_{s}{ }^{\prime}$ must be sufficiently small to keep the approximation valid. Note also that for a fixed turbid medium the ratio between the API and the DA depends slowly on $r$, the dominant effect being almost a constant shift.

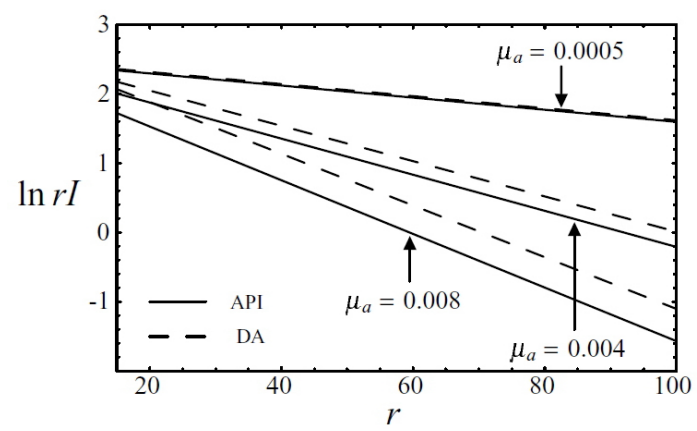

Figure 9: Plots of $\ln [r I(r)]$ for $\mu_{s}^{\prime}=0.05 \mathrm{~mm}^{-1} . \quad I$ is measured in $\mathrm{mm}^{-2} \mathrm{ps}^{-1}, \mu_{a}$ in $\mathrm{mm}^{-1}$ and $r$ in $\mathrm{mm}$.

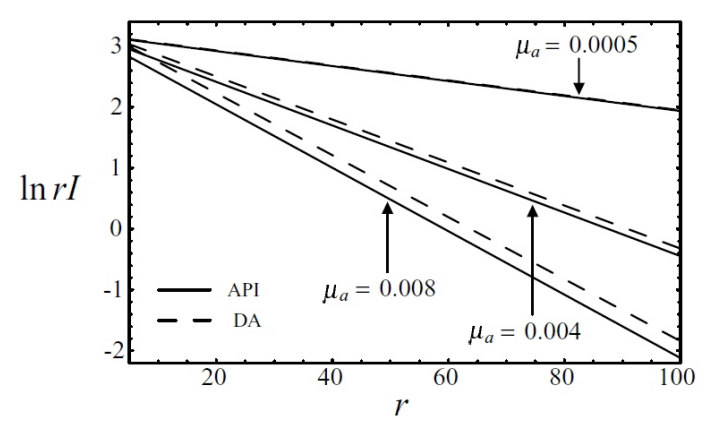

Figure 10: Plots of $\ln [r I(r)]$ for $\mu_{s}^{\prime}=$ $0.1 \mathrm{~mm}^{-1}$. $I$ is measured in $\mathrm{mm}^{-2} \mathrm{ps}^{-1}$, $\mu_{a}$ in $\mathrm{mm}^{-1}$ and $r$ in $\mathrm{mm}$.

\subsection{Semi-infinite medium}

A semi-infinite medium is an infinite region with a single boundary plane located at $z=0$. For simplicity to determine the fluence rate we apply the method of images to ensure that it satisfies a Dirichlet boundary condition at $z=0, I(x, y, 0, t)=0$ [6]. The image sources are placed at $z= \pm z_{0}$, where $z_{0}=1 / \mu_{s}^{\prime}$ is the $z$ coordinate of the point where the photons are assumed to be initially scattered. The image source distribution is then given by

$$
S(\mathbf{r}, t)=S \delta(x) \delta(y)\left[\delta\left(z-z_{0}\right)-\delta\left(z+z_{0}\right)\right] \theta(t) .
$$

Note that this approximated framework is only expected to be valid if measurements are made at distances much larger than $z_{0}$ and than an eventual boundary extrapolation length. In this circunstances, uncertainties in the value of $z_{0}$ and corrections due to the extrapolated boundary length should be negligible [6].

Applying the convolution (13) the fluence rate satisfying the Dirichlet boundary condition is given by

$$
I(\rho, z, t)=I_{-}(\rho, z, t)-I_{+}(\rho, z, t)
$$

with

$$
I_{ \pm}(\rho, z, t)=\frac{S}{2 \pi v^{2}} \int_{0}^{t-\sqrt{\left[\left(z \pm z_{0}\right)^{2}+\rho^{2}\right] / v^{2}}} d \tau \frac{(t-\tau)^{-3}}{B\left[\frac{3}{2}, \frac{v(t-\tau)}{4 D}+1\right]}\left[1-\frac{\left(z \pm z_{0}\right)^{2}+\rho^{2}}{v^{2}(t-\tau)^{2}}\right]^{\frac{v(t-\tau)}{4 D}}
$$




$$
\times \exp \left[-v \mu_{a}(t-\tau)\right]
$$

where in cylindrical coordinates $\rho=\sqrt{x^{2}+y^{2}}$. Taking as before $D<<r, r<<v t$, $v t>>r^{2} / D$ and $D \mu_{a}<<1$ to expand around the diffusion limit we are lead to

$$
I_{ \pm}(\rho, z)=I_{ \pm}^{\mathrm{DA}}(\rho, z)\left[1-r_{ \pm} \sqrt{D} \mu_{a}^{3 / 2}-6 D \mu_{a}+\frac{1}{2} r_{ \pm}^{2} D \mu_{a}^{3}+\mathcal{O}\left(\varepsilon^{3 / 2}\right)\right]
$$

where $r_{ \pm}=\sqrt{\left(z \pm z_{0}\right)^{2}+\rho^{2}}$ and

$$
I_{ \pm}^{\mathrm{DA}}(\rho, z)=\frac{S}{4 \pi D r_{ \pm}} \exp \left(-r_{ \pm} \sqrt{\frac{\mu_{a}}{D}}\right)
$$

are the contributions of the DA.

In the infinite half-space the experimentally relevant quantity is the reflectance $R(\rho, t)$ defined as the amplitude of the photon current $\mathbf{J}(\rho, z, t)$ at the boundary plane $z=0, R(\rho, t)=|\mathbf{J}(\rho, 0, t)|[6]$. This current is determined using Fick's law,

$$
\mathbf{J}(\rho, z, t)=-D \nabla I(\rho, z, t) .
$$

Calculating the gradient of the fluence rate we find

$$
\mathbf{J}(\rho, 0)=\mathbf{J}^{\mathrm{DA}}(\rho, 0)\left[1-6 D \mu_{a}-\frac{r_{0}^{2} \mu_{a}^{2}}{1+r_{0} \sqrt{\mu_{a} / D}}\left(1-\frac{1}{2} r_{0} \sqrt{D} \mu_{a}^{3 / 2}\right)+\mathcal{O}\left(\varepsilon^{3 / 2}\right)\right]
$$

where $r_{0}=\sqrt{\rho^{2}+z_{0}^{2}}$ and the diffusion limit contribution is

$$
\mathbf{J}^{\mathrm{DA}}(\rho, 0)=-\frac{S \mathbf{z}_{0}}{2 \pi r_{0}^{3}} \exp \left(-r_{0} \sqrt{\frac{\mu_{a}}{D}}\right)\left(1+r_{0} \sqrt{\frac{\mu_{a}}{D}}\right)
$$

with $\mathbf{z}_{0}=\left(0,0, z_{0}\right)$. Consequently, the reflectance is given by

$$
R(\rho)=R^{\mathrm{DA}}(\rho)\left[1-6 D \mu_{a}-\frac{r_{0}^{2} \mu_{a}^{2}}{1+r_{0} \sqrt{\mu_{a} / D}}\left(1-\frac{1}{2} r_{0} \sqrt{D} \mu_{a}^{3 / 2}\right)+\mathcal{O}\left(\varepsilon^{3 / 2}\right)\right],
$$

where

$$
R^{\mathrm{DA}}(\rho)=\frac{S z_{0}}{2 \pi r_{0}^{3}} \exp \left(-r_{0} \sqrt{\frac{\mu_{a}}{D}}\right)\left(1+r_{0} \sqrt{\frac{\mu_{a}}{D}}\right) .
$$

To compare with the DA it is convenient to plot $\ln \left[r_{0}^{3} R(\rho)\right]$ as we do in figures 11 and 12 for $S=100 \mathrm{ps}^{-1}$. Just like for the unbounded infinite space, non-diffusive effects are enhanced for decreasing reduced scattering coefficients $\mu_{s}{ }^{\prime}$ and increasing absorption coefficients $\mu_{a}$. 


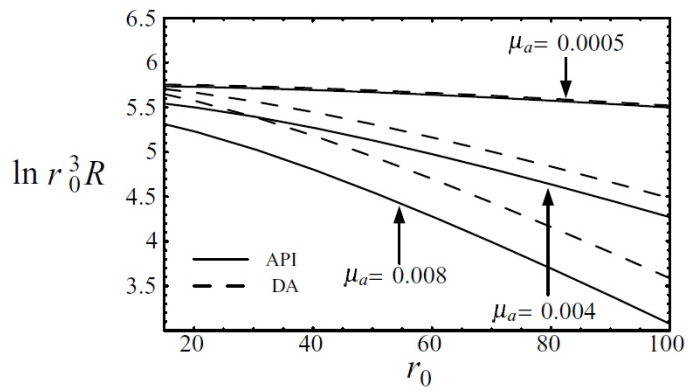

Figure 11: Plots of $\ln \left[r_{0}^{3} R(\rho)\right]$ for $\mu_{s}^{\prime}=0.05 \mathrm{~mm}^{-1} . \quad R$ is measured in $\mathrm{mm}^{-2} \mathrm{ps}^{-1}, \mu_{a}$ in $\mathrm{mm}^{-1}$ and $r_{0}$ in $\mathrm{mm}$.

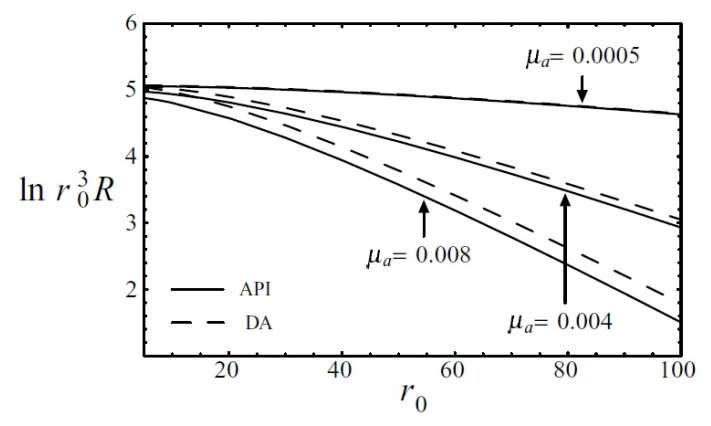

Figure 12: Plots of $\ln \left[r_{0}^{3} R(\rho)\right]$ for $\mu_{s}^{\prime}=$ $0.1 \mathrm{~mm}^{-1}$. $R$ is measured in $\mathrm{mm}^{-2} \mathrm{ps}^{-1}$, $\mu_{a}$ in $\mathrm{mm}^{-1}$ and $r_{0}$ in $\mathrm{mm}$.

\subsection{Infinite slab}

The analysis applied to the semi-infinite medium may be generalized to the infinite slab geometry [6]. In this configuration there are two plane boundaries located at $z=0$ and $z=d$, where $d$ is the thickness of the slab. The solution to this problem is obtained considering an infinite number of dipole sources placed at regular intervals in the $z$ axis, $z=2 j d \pm z_{0}$, where $j=0, \pm 1, \pm 2, \cdots$. The continuous wave source distribution is thus

$$
S(\mathbf{r}, t)=S \delta(x) \delta(y) \sum_{j=-\infty}^{+\infty}\left[\delta\left(z-2 j d-z_{0}\right)-\delta\left(z-2 j d+z_{0}\right)\right] \theta(t) .
$$

The fluence rate satisfying Dirichlet boundary conditions at $z=0$ and $z=d$ is then given by

$$
I(\rho, z, t)=\sum_{j=-\infty}^{+\infty}\left[I_{-j}(\rho, z, t)-I_{+j}(\rho, z, t)\right]
$$

with

$$
\begin{aligned}
I_{ \pm j}(\rho, z, t)=\frac{S}{2 \pi v^{2}} \int_{0}^{t-\sqrt{\left[\left(z-2 j d \pm z_{0}\right)^{2}+\rho^{2}\right] / v^{2}}} d \tau \frac{(t-\tau)^{-3}}{B\left[\frac{3}{2}, \frac{v(t-\tau)}{4 D}+1\right]} \exp \left[-v \mu_{a}(t-\tau)\right] \\
\quad \times\left[1-\frac{\left(z-2 j d \pm z_{0}\right)^{2}+\rho^{2}}{v^{2}(t-\tau)^{2}}\right]^{\frac{v(t-\tau)}{4 D}} .
\end{aligned}
$$


The corresponding time independent expansion around the diffusion phase point is

$$
I_{ \pm j}(\rho)=I_{ \pm j}^{\mathrm{DA}}(\rho)\left[1-r_{ \pm j} \sqrt{D} \mu_{a}^{3 / 2}-6 D \mu_{a}+\frac{1}{2} r_{ \pm j}^{2} D \mu_{a}^{3}+\mathcal{O}\left(\varepsilon^{3 / 2}\right)\right]
$$

where

$$
I_{ \pm j}^{\mathrm{DA}}(\rho)=\frac{S}{4 \pi D r_{ \pm j}} \exp \left(-r_{ \pm j} \sqrt{\frac{\mu_{a}}{D}}\right)
$$

are the DA contributions and $r_{ \pm j}=\sqrt{\left(z-2 j d \pm z_{0}\right)^{2}+\rho^{2}}$.

The experimentally relevant quantities are the reflectance $R(\rho, t)$ and the transmittance $T(\rho, t)$ defined as the amplitudes of the photon current $\mathbf{J}(\rho, z, t)$, respectively, at $z=0$ and $z=d, R(\rho, t)=|\mathbf{J}(\rho, 0, t)|$ and $T(\rho, t)=|\mathbf{J}(\rho, d, t)|$. Let us first consider the reflected photon current $\mathbf{J}(\rho, 0, t)$. Evaluation of the fluence rate gradient leads to

$$
\begin{aligned}
\mathbf{J}(\rho, 0)=\sum_{j=-\infty}^{+\infty} \mathbf{J}_{j}^{\mathrm{DA}}(\rho, 0) & {\left[1-\frac{r_{0 j}^{2} \mu_{a}^{2}}{1+r_{0 j} \sqrt{\mu_{a} / D}}\left(1-\frac{1}{2} r_{0 j} \sqrt{D} \mu_{a}^{3 / 2}\right)\right.} \\
- & \left.6 D \mu_{a}+\mathcal{O}\left(\varepsilon^{3 / 2}\right)\right]
\end{aligned}
$$

where $r_{0 j}=\sqrt{\rho^{2}+\left(2 j d+z_{0}\right)^{2}}$ and the diffusion limit contributions to the infinite sum are

$$
\mathbf{J}_{j}^{\mathrm{DA}}(\rho, 0)=-\frac{S \mathbf{z}_{0 j}}{2 \pi r_{0 j}^{3}} \exp \left(-r_{0 j} \sqrt{\frac{\mu_{a}}{D}}\right)\left(1+r_{0 j} \sqrt{\frac{\mu_{a}}{D}}\right),
$$

with $\mathbf{z}_{0 j}=\left(0,0,2 j d+z_{0}\right)$. Since $\mathbf{J}(\rho, 0)$ is a real vector for which only the $z$ component $J_{z}(\rho, 0)$ is non-zero the reflectance is simply equal to

$$
\begin{aligned}
R(\rho)=\sum_{j=-\infty}^{+\infty} R_{j}^{\mathrm{DA}}(\rho) & {\left[1-\frac{r_{0 j}^{2} \mu_{a}^{2}}{1+r_{0 j} \sqrt{\mu_{a} / D}}\left(1-\frac{1}{2} r_{0 j} \sqrt{D} \mu_{a}^{3 / 2}\right)\right.} \\
& \left.-6 D \mu_{a}+\mathcal{O}\left(\varepsilon^{3 / 2}\right)\right],
\end{aligned}
$$

where the diffusion limit contributions are

$$
R_{j}^{\mathrm{DA}}(\rho)=\frac{S z_{0 j}}{2 \pi r_{0 j}^{3}} \exp \left(-r_{0 j} \sqrt{\frac{\mu_{a}}{D}}\right)\left(1+r_{0 j} \sqrt{\frac{\mu_{a}}{D}}\right),
$$

with $z_{0 j}=2 j d+z_{0}$. The infinite half-space result is given by the $j=0$ term, naturally obtained in the limit $d \rightarrow+\infty$. In figures $13-16$ we present plots of $\ln \left[\rho^{3} R(\rho)\right]$ for $S=100 \mathrm{ps}^{-1}$. The results for fixed $d$ (see figures 13 and 14) again show that non-diffusive effects increase with the absorption coeficient $\mu_{a}$ and when 
the reduced scattering coefficient $\mu_{s}^{\prime}$ diminishes. On the other hand figures 13,15 and 16 show that the isotropic non-diffusive effects increase with $d$.

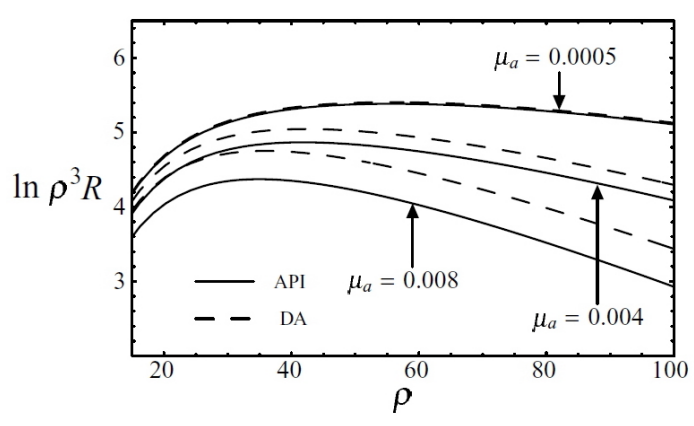

Figure 13: Plots of $\ln \left[\rho^{3} R(\rho)\right]$ for $d=$ $100 \mathrm{~mm}, \mu_{s}^{\prime}=0.05 \mathrm{~mm}^{-1}$. $R$ is measured in $\mathrm{mm}^{-2} \mathrm{ps}^{-1}, \mu_{a}$ in $\mathrm{mm}^{-1}$ and $\rho$ in $\mathrm{mm}$.

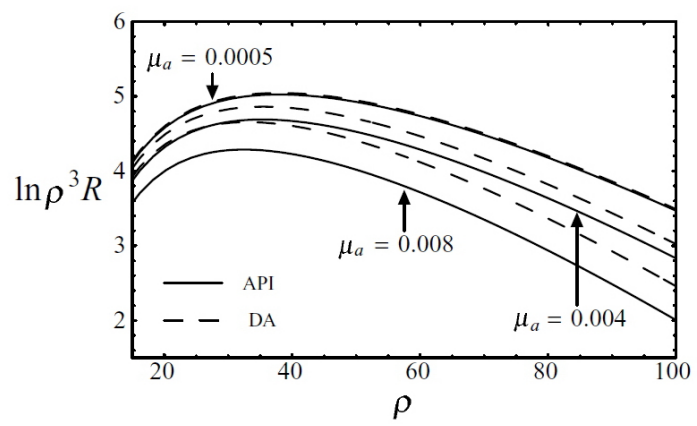

Figure 15: Plots of $\ln \left[\rho^{3} R(\rho)\right]$ for $d=$ $50 \mathrm{~mm}, \mu_{s}^{\prime}=0.05 \mathrm{~mm}^{-1}$. $R$ is measured in $\mathrm{mm}^{-2} \mathrm{ps}^{-1}, \mu_{a}$ in $\mathrm{mm}^{-1}$ and $\rho$ in $\mathrm{mm}$.

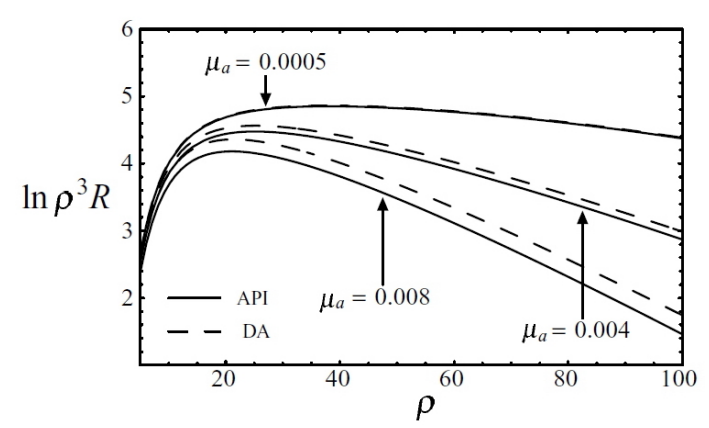

Figure 14: Plots of $\ln \left[\rho^{3} R(\rho)\right]$ for $d=$ $100 \mathrm{~mm}, \mu_{s}^{\prime}=0.1 \mathrm{~mm}^{-1}$. $R$ is measured in $\mathrm{mm}^{-2} \mathrm{ps}^{-1}, \mu_{a}$ in $\mathrm{mm}^{-1}$ and $\rho$ in $\mathrm{mm}$.

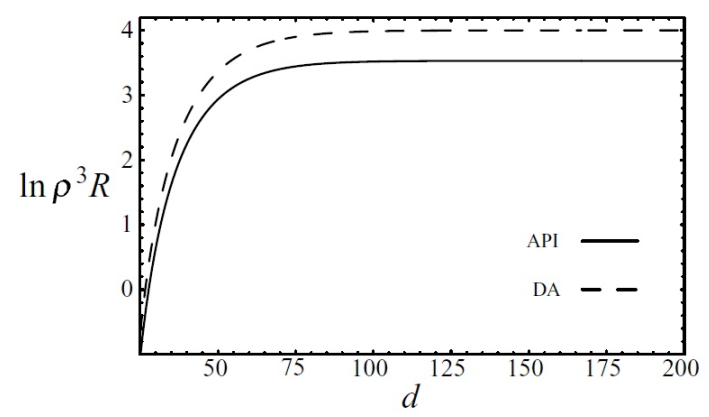

Figure 16: Plots of $\ln \left[\rho^{3} R(\rho)\right]$ for $\rho=80 \mathrm{~mm}, \mu_{s}^{\prime}=0.05 \mathrm{~mm}^{-1}$ and $\mu_{a}=0.008 \mathrm{~mm}^{-1}$. $R$ is measured in $\mathrm{mm}^{-2} \mathrm{ps}^{-1}$ and $d$ in $\mathrm{mm}$.

Similarly, rearraging the terms in the infinite sum, we find for the transmitted current the following expression,

$$
\mathbf{J}(\rho, d)=\sum_{j=-\infty}^{+\infty} \mathbf{J}_{j}^{\mathrm{DA}}(\rho, d)\left[1-\frac{\hat{r}_{0 j}^{2} \mu_{a}^{2}}{1+\hat{r}_{0 j} \sqrt{\mu_{a} / D}}\left(1-\frac{1}{2} \hat{r}_{0 j} \sqrt{D} \mu_{a}^{3 / 2}\right)\right.
$$




$$
\left.-6 D \mu_{a}+\mathcal{O}\left(\varepsilon^{3 / 2}\right)\right]
$$

where now $\hat{r}_{0 j}=\sqrt{\rho^{2}+\left[d(2 j-1)+z_{0}\right]^{2}}$ and the diffusion limit contributions are

$$
\mathbf{J}_{j}^{\mathrm{DA}}(\rho, d)=\frac{S \hat{\mathbf{z}}_{0 j}}{2 \pi \hat{r}_{0 j}^{3}} \exp \left(-\hat{r}_{0 j} \sqrt{\frac{\mu_{a}}{D}}\right)\left(1+\hat{r}_{0 j} \sqrt{\frac{\mu_{a}}{D}}\right),
$$

with $\hat{\mathbf{z}}_{0 j}=\left(0,0, d(1-2 j)-z_{0}\right)$. The transmittance is then given by

$$
\begin{aligned}
T(\rho)=\sum_{j=-\infty}^{+\infty} T_{j}^{\mathrm{DA}}(\rho) & {\left[1-\frac{\hat{r}_{0 j}^{2} \mu_{a}^{2}}{1+\hat{r}_{0 j} \sqrt{\mu_{a} / D}}\left(1-\frac{1}{2} \hat{r}_{0 j} \sqrt{D} \mu_{a}^{3 / 2}\right)\right.} \\
& \left.-6 D \mu_{a}+\mathcal{O}\left(\varepsilon^{3 / 2}\right)\right]
\end{aligned}
$$

where the diffusion limit contributions are

$$
T_{j}^{\mathrm{DA}}(\rho)=\frac{S \hat{z}_{0 j}}{2 \pi \hat{r}_{0 j}^{3}} \exp \left(-\hat{r}_{0 j} \sqrt{\frac{\mu_{a}}{D}}\right)\left(1+\hat{r}_{0 j} \sqrt{\frac{\mu_{a}}{D}}\right),
$$

with $\hat{z}_{0 j}=d(1-2 j)-z_{0}$. Clearly, $T(\rho) \rightarrow 0$ as $d \rightarrow+\infty$, as it should be for the semiinfinite medium. In practice, it is suficiently accurate to consider a finite number of terms in the fluence rate solution. Such number depends on the specific experimental setup [6]. In our calculations we have considered $|j| \leq 5$ for the reflectance and $|j| \leq 10$ for the transmittance. Transmittance results and comparison with the diffusion approximation are presented in figures 17 to 20 where $S=100 \mathrm{ps}^{-1}$. These figures confirm that non-diffusive isotropic effects increase with $\mu_{a}, 1 / \mu_{s}{ }^{\prime}$ and $d$.

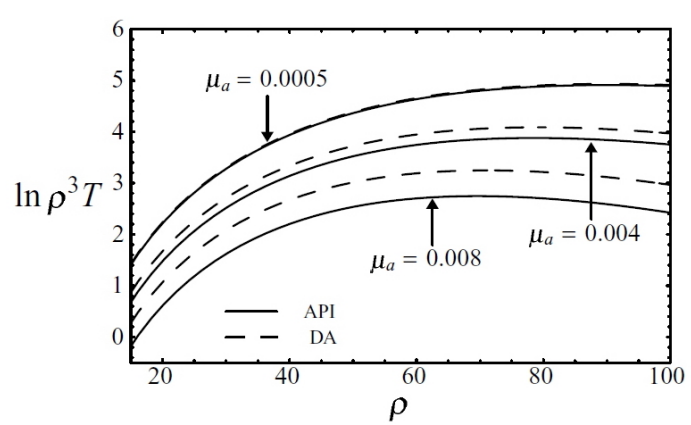

Figure 17: Plots of $\ln \left[\rho^{3} T(\rho)\right]$ for $d=$ $100 \mathrm{~mm}, \mu_{s}^{\prime}=0.05 \mathrm{~mm}^{-1}$. $T$ is measured in $\mathrm{mm}^{-2} \mathrm{ps}^{-1}, \mu_{a}$ in $\mathrm{mm}^{-1}$ and $\rho$ in $\mathrm{mm}$.

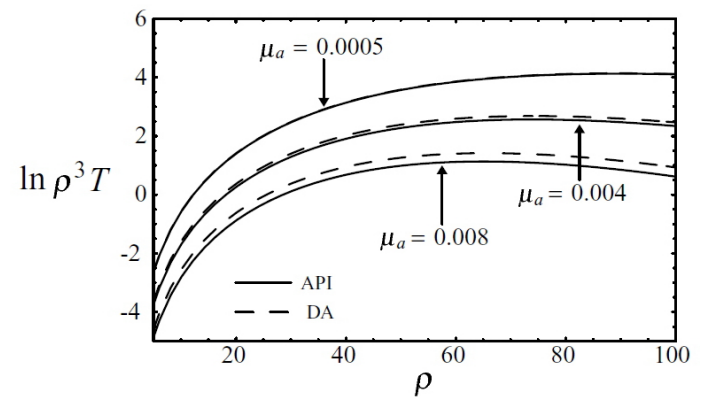

Figure 18: Plots of $\ln \left[\rho^{3} T(\rho)\right]$ for $d=$ $100 \mathrm{~mm}, \mu_{s}^{\prime}=0.1 \mathrm{~mm}^{-1}$. $T$ is measured in $\mathrm{mm}^{-2} \mathrm{ps}^{-1}, \mu_{a}$ in $\mathrm{mm}^{-1}$ and $\rho$ in $\mathrm{mm}$. 


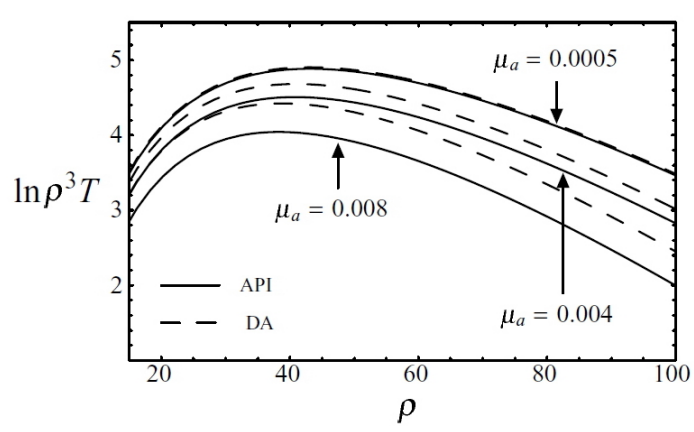

Figure 19: Plots of $\ln \left[\rho^{3} T(\rho)\right]$ for $d=$ $50 \mathrm{~mm}, \mu_{s}^{\prime}=0.05 \mathrm{~mm}^{-1}$. $T$ is measured in $\mathrm{mm}^{-2} \mathrm{ps}^{-1}, \mu_{a}$ in $\mathrm{mm}^{-1}$ and $\rho$ in $\mathrm{mm}$.

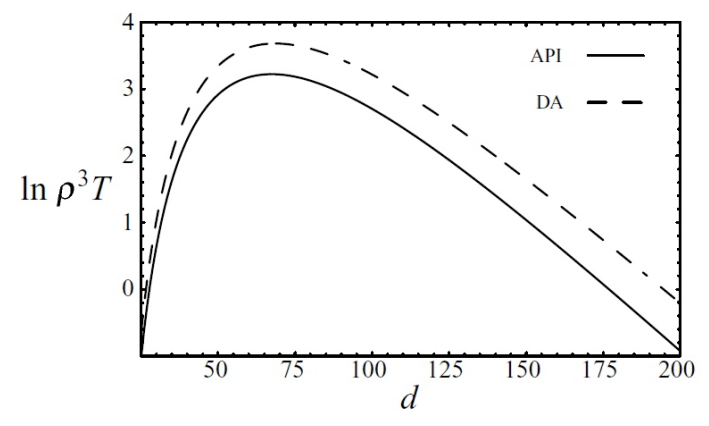

Figure 20: Plots of $\ln \left[\rho^{3} T(\rho)\right]$ for $\rho=80 \mathrm{~mm}, \mu_{s}^{\prime}=0.05 \mathrm{~mm}^{-1}$ and $\mu_{a}=0.008 \mathrm{~mm}^{-1}$. $T$ is measured in $\mathrm{mm}^{-2} \mathrm{ps}^{-1}$ and $d$ in $\mathrm{mm}$.

\section{Conclusions}

In this paper we have considered the application of the path integral (PI) approach to describe the propagation of photons in homogeneous turbid media characterized by an absorption coefficient $\mu_{a}$, a reduced scattering coefficient $\mu_{s}^{\prime}$ and a refractive index $n$. We have extended the time domain analysis [18]-[22] to include more general source functions and introduced a new analytic perturbative method to determine the PI photon propagator, the root object needed to define relevant physical quantities required for non-invasive measurements of tissue optical properties. Working for simplicity in the isotropic regime, we have determined a new analytic expansion for the PI propagator centered on the diffusion limit. We have shown that this expansion defines new approximated PI propagators which relative to the diffusion approximation (DA) lead to improved descriptions of photon propagation in turbid media.

To illustrate the application of this approach we have considered the example of continuous wave systems designed for optical tomography. We have determined new analytic solutions for the continuous wave fluence rate in three representative geometric configurations: the infinite unbounded medium, the semi-infinite medium and the infinite slab. For the latter two geometries we have calculated the corresponding reflectance and transmittance functions. Our method allows a direct comparison with the DA, the center of the perturbative expansion. Based on such a comparative analysis we have shown that non-diffusive effects are enhanced in 
turbid regions with increasing absorption coefficients and decreasing reduced scattering coefficients as well as when the distance between boundaries increases. In a forthcoming work we will also analyse frequency domain systems and compare with experimental results.

\section{Acknowledgements}

We are grateful for the financial support of Centro de Electrónica, Optoelectrónica e Telecomunicações (CEOT) and of project POCTI/FAT/42850/2001 financed by the Fundação para a Ciência e a Tecnologia (FCT) and Fundo Social Europeu (FSE). RN also acknowledges the support of Centro Multidisciplinar de Astrofísica (CENTRA).

\section{References}

[1] S. Chandrasekhar, Radiative Transfer, Oxford University Press, London, 1950.

[2] A. Ishimaru, Wave Propagation and Scattering in Random Media, Academic Press, New York, 1978.

[3] M. Patterson, B. Wilson and D. Wyman, The propagation of optical radiation in tissue: I. Models of radiation transport and their application, Lasers Med. Sci. 6 (1992), pp. 155-168.

[4] A. Yodh and B. Chance, Spectroscopy and imaging with diffusing light, Phys. Today 48 (1995), pp. 34-40.

[5] A. Gibson, J. Hebden and S. Arridge, Recent advances in diffuse optical imaging, Phys. Med. Biol. 50 (2005),pp. R1-R43.

[6] M. Patterson, B. Chance and B. Wilson, Time resolved reflectance and transmittance for the noninvasive measurement of tissue optical properties, Appl. Opt. 28 (1989), pp. 2331-2336.

[7] A. Ishimaru, Correlation functions of a wave in a random distribution of stationary and moving scatterers, Radio Sci. 10 (1975), pp. 4552.

[8] B. Beauvoit, H. Liu, K. Kang, P. D. Kaplan, M. Miwa, and B. Chance, Characterization of absorption and scattering properties of various yeast strains by time-resolved spectroscopy, Cell Biophys. 23, (1993), pp. 91109.

[9] D. Passos, J. C. Hebden, P. N. Pinto and R. Guerra, A tissue phantom for optical diagnostics based on a suspension of microspheres with a fractal size distribution, J. Biomedical Optics 10 (2005): art. no. 064036. 
[10] J. Fishkin and E. Gratton, Propagation of photon-density waves in strongly scattering media containing an absorbing semi-infinite plane bounded by a straight edge, J. Opt. Soc. Am. A 10 (1993), pp. 127-140.

[11] R. Haskell, L. Svaasand, T.-T.Tsay, T.-C. Feng, M. McAdams and B. Tromberg, Boundary conditions for the diffusion equation in radiative transfer, J. Opt. Soc. Am. A 11 (1994), pp. 2727-2741.

[12] J. Hebden, S. Arridge and D. Delpy, Optical imaging in medicine: I. Experimental techniques, Phys. Med. Biol. 42 (1997), pp. 825-840.

[13] S. Arridge and J. Hebden, Optical imaging in medicine: II. Modelling and reconstruction, Phys. Med. Biol. 42 (1997), pp. 841-853.

[14] S. Arridge and M. Schweiger, Image reconstruction in optical tomography, Phil. Trans. R. Soc. Lond. B 352 (1997), pp. 717-726.

[15] S. Arridge, Optical tomography in medical imaging, Inverse Problems 15 (1999), pp. R41-R93.

[16] J. Tessendorf, Radiative transfer as a sum of paths, Phys. Rev. A 35 (1987), pp. $872-878$.

[17] J. Schotland, J. Haselgrove and J. Leigh, Photon hitting density, Appl. Opt. 32 (1993), pp. 448-453.

[18] L. Perelman, J. Wu, I. Itzkan and M. Feld, Photon migration in turbid media using path integrals, Phys. Rev. Lett. 72 (1994), pp. 1341-1344.

[19] L. Perelman, J. Wu, Y. Wang, I. Itzkan, R. Dasari and M. Feld, Time-dependent photon migration using path integrals, Phys. Rev. E 51 (1995), pp. 6134-6141.

[20] A. Polishchuk, M. Zevallos, F. Liu and R. Alfano, Generalization of Fermats principle for photons in random media: the least mean square curvature of paths and photon diffusion on the velocity sphere, Phys. Rev. E 53 (1996), pp. 5523-5526.

[21] L. Perelman, J. Winn, J. Wu, Y. Wang, R. Dasari and M. Feld, Photon migration of near-diffusive photons in turbid media: a Lagrangian-based approach, J. Opt. Soc. Am. A 14 (1997), pp. 224-229.

[22] J. Winn, L. Perelman, K. Chen, J. Wu, R. Dasari and M. Feld, Distribution of the paths of early-arriving photons traversing a turbid medium, Appl. Opt. 37 (1998), pp. 8085-8091. 
[23] R. Feynman and A. Hibbs, Quantum Mechanics and Path Integrals, McGrawHill, New York, 1965.

[24] N. Lebedev, Special Functions and their Applications, Dover, New York, 1972.

[25] I. Gradshteyn and I. Ryzhik, Tables of Integrals, Series and Products, edited by A. Jeffrey and D. Zwillinger, Academic Press, London, 2000.

[26] V. Twersky, On propagation in random media of discrete scatterers, Proc. Am. Math. Soc. Symp. Stochas. Proc. Math. Phys. Eng. 16 (1964), pp. 84-116.

[27] V. Twersky, Theory of microwave measurements on higher statistical moments of randomly scattered fields, in Electromagnetic Scattering, R. L. Rowell and R. S. Stein, eds., Gordon and Breach, New York, 1967, pp. 579-695.

[28] C. I. Beard, T. H. Kays and V. Twerski, Scattering by random distribution of spheres vs. concentration, IEEE Trans. Ant. Prop. AP-15 (1967), pp. 99-118.

[29] Y. N. Barabanenkov, On the spectral theory of radiation transport equations, Sov. Phys. JETP 26 (1969), pp. 679-684.

\section{Appendices A: Derivation of the transport equation from the multiple scattering theory}

\section{A.1. Fundamental equations}

In this appendix we derive the Boltzmann transfer equation from the multiple scattering theory. Introductory considerations have already been made in the second to the fifth paragraphs of the Introduction and will not be repeated here.

Consider a random distribution of $N$ particles (not necessarily identical) located at $\mathbf{r}_{1}, \mathbf{r}_{2}, \cdots, \mathbf{r}_{N}$ in a volume $V$. We consider a scalar field $\psi^{a}$ at a point $\mathbf{r}_{a}$ between the scatterers. This field may be one of the rectangular components of the electric or magnetic field and satisfies the wave equation $\left(\nabla+k^{2}\right) \psi=0$, where $k=2 \pi / \lambda$ is the wave number in the medium surrounding the particles.

Ishimaru [7] has derived the transport equation from the Twersky equation [26, 27]:

$$
\left\langle\psi^{a} \psi^{b *}\right\rangle=\left\langle\psi^{a}\right\rangle\left\langle\psi^{b *}\right\rangle+\int v_{s}^{a} v_{s}^{b *}\left\langle\left|\psi^{s}\right|^{2}\right\rangle \rho\left(\mathbf{r}_{s}\right) d^{3} \mathbf{r}_{s}
$$

Here $\langle\cdot\rangle$ means ensemble average, $\rho\left(\mathbf{r}_{s}\right)$ is the density and $v_{s}^{a}$ satisfies the integral equation 


$$
v_{s}^{a}=u_{s}^{a}+\int u_{t}^{a} v_{s}^{t} \rho\left(\mathbf{r}_{t}\right) d^{3} \mathbf{r}_{t}
$$

In this equation $u_{s}^{a}$ is the single scattering operator that outputs the scattered field $U_{s}^{a}$ at $\mathbf{r}_{a}$, due the scatterer at $\mathbf{r}_{s}$, when the wave $\Psi^{s}$ is incident on it: $U_{a}^{a}=u_{s}^{a} \Psi^{s}$. The general convention is that the superscript refers to the observation point and the subscript to the emission point. The Twersky equation (59) is obtained from an averaging procedure of an integral equation approximation for the scattered field. One of the underlying assumptions is that the particles location are independent of each other, so that the joint probability of finding particle 1 at $\mathbf{r}_{1}, \cdots$, particle $N$ at $\mathbf{r}_{N}$ may be factorized:

$$
W\left(\mathbf{r}_{1}, \cdots, \mathbf{r}_{N}\right)=w\left(\mathbf{r}_{1}\right) \times \cdots \times w\left(\mathbf{r}_{N}\right) .
$$

This assumption is justified for systems where the density is low and the particle size is much smaller than the average inter-particle distance. A typical system where this is a good assumption is a sparse suspension of particles. However, for biological systems the density is high and the distance between scatterers may be of the order of magnitude of their size. The main contribution to scattering is thought to come from the cell mitochondria, but also from the cell nucleus, the cell walls and the other organelles [8]. In this case the position of the scatterers is not independent from each other and it is appropriate to introduce a pair distribution function

$$
P_{s t}=\frac{g\left(\mathbf{r}_{s}, \mathbf{r}_{t}\right)}{V^{2}}
$$

where $g\left(\mathbf{r}_{s}, \mathbf{r}_{t}\right) / V$ is the conditional probability that given scatterer $s$ at $\mathbf{r}_{s}$, we find scatterer $t$ at $\mathbf{r}_{t}$ and $V$ is the volume of the system.

The question that arises naturally is to know if a transport equation can be derived under these circumstances. In this appendix we generalize Ishimarus derivation of the transport equation to the case of dense systems with correlated scatterers. To do that we follow the heuristic prescription of Beard [28] to include the pair distribution function in the Twersky equation:

$$
\left\langle\psi^{a} \psi^{b *}\right\rangle=\left\langle\psi^{a}\right\rangle\left\langle\psi^{b *}\right\rangle+\rho \int v_{s}^{a} v_{s}^{b *}\left\langle\left|\psi^{s}\right|^{2}\right\rangle d^{3} \mathbf{r}_{s}+\rho^{2} \iint v_{s}^{a} v_{s}^{b *}\left\langle\psi^{s} \psi^{t *}\right\rangle G\left(\mathbf{r}_{s}, \mathbf{r}_{t}\right) d^{3} \mathbf{r}_{s} d^{3} \mathbf{r}_{t},
$$

with 


$$
G\left(\mathbf{r}_{s}, \mathbf{r}_{t}\right)=1-\sum_{n} \sum_{m \neq n} P_{s t} / \rho^{2}=1-g\left(\mathbf{r}_{s}, \mathbf{r}_{t}\right)(N-1) / N,
$$

where the summation is made on the particles, $N$ is the total number of particles and we have assumed a constant density $\rho$.

In the next section we present the original derivation of Ishimaru, starting from (59), and emphasizing the conditions under which the derivation is made. In the last section we extend the reasoning to the case of dense systems, by applying (63).

\section{A.2. Derivation of the transport equation for independent scatterers}

The geometry for the calculations presented in this section are depicted in Figure 21. Ishimaru's derivation of the transport equation is based on a series of approximations. The first one is to assume that the single scattering operator is given by the far field approximation:

$$
u_{s}^{a}=f\left(\hat{\mathbf{s}}_{s-a}, \hat{\mathbf{s}}_{s}\right) \frac{e^{i k\left|\mathbf{r}_{a}-\mathbf{r}_{s}\right|}}{\left|\mathbf{r}_{a}-\mathbf{r}_{s}\right|},
$$

where $f\left(\hat{\mathbf{s}}, \hat{\mathbf{s}}^{\prime}\right)$ is the scattering amplitude of the scatterer $\left(\int_{4 \pi}|f|^{2} d \Omega=\sigma_{s}\right.$, the scattering cross-section), $\hat{\mathbf{s}}_{s-a}$ is the unit vector in the direction of $\mathbf{r}_{s}$ to $\mathbf{r}_{a}$ (that is, in the direction of $\mathbf{r}_{a}-\mathbf{r}_{s}$ ) and $\hat{\mathbf{s}}_{s}$ means the unit vector in the direction of the incident field at point $\mathbf{r}_{s}$. This is only true if the distance between scatterers is much larger than the wavelength. It is then possible to show that $v_{s}^{a}$ has approximately the same form:

$$
v_{s}^{a}=f\left(\hat{\mathbf{s}}_{s-a}, \hat{\mathbf{s}}_{s}\right) \frac{e^{i K\left|\mathbf{r}_{a}-\mathbf{r}_{s}\right|}}{\left|\mathbf{r}_{a}-\mathbf{r}_{s}\right|},
$$

with $K=k+2 \pi \rho f(0) / k, f(0)=f\left(\hat{\mathbf{s}}_{s}, \hat{\mathbf{s}}_{s}\right)$.

The link between the Twersky equation and the transport equation is done through the relation between the correlation function and the specific intensity $I(\mathbf{r}, \hat{\mathbf{s}})$ :

$$
\left\langle\psi\left(\mathbf{r}_{a}\right) \psi^{*}\left(\mathbf{r}_{b}\right)\right\rangle \approx \int I\left(\mathbf{r}_{c a b}, \hat{\mathbf{s}}_{c a b}\right) e^{i K_{r} \hat{\mathbf{s}}_{c a b} \cdot \mathbf{r}_{d a b}} d \hat{\mathbf{s}}_{c a b} .
$$

In the following we will adopt the following convention, in order to make the expressions self-readable: the subscript $c$ denotes a centre point and the subscript $d$ a difference point. Accordingly, in this expression $\mathbf{r}_{c a b}=\left(\mathbf{r}_{a}+\mathbf{r}_{b}\right) / 2, \mathbf{r}_{d a b}=\mathbf{r}_{a}-\mathbf{r}_{b}$, $\int d \hat{\mathbf{s}}_{c a b}$ means angular integration on all directions $\hat{\mathbf{s}}_{c a b}$ centred on point $\mathbf{r}_{c a b}$ and $K_{r}$ 
is the real part of $K$. Expression (67) is valid when the correlation function is a slowly varying function of $\mathbf{r}[29]$, specifically, it must vary slowly over the correlation distance of the field. This is the second assumption implicit in Ishimaru's derivation. The coherent intensity is defined in the same way:

$$
\left\langle\psi\left(\mathbf{r}_{a}\right)\right\rangle\left\langle\psi^{*}\left(\mathbf{r}_{b}\right)\right\rangle \approx \int I_{c}\left(\mathbf{r}_{c a b}, \hat{\mathbf{s}}_{c a b}\right) e^{i K_{r} \hat{\mathbf{s}}_{c a b} \cdot \mathbf{r}_{d a b}} d \hat{\mathbf{s}}_{c a b} .
$$
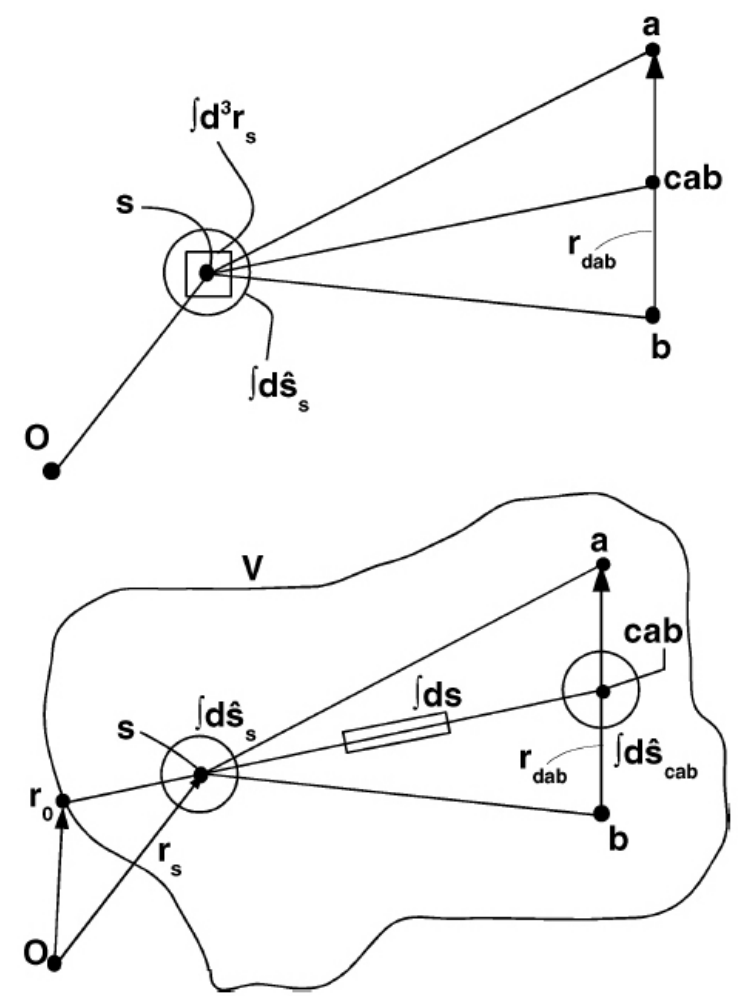

Figure 21: Geometry used in Section A.2. A square around a given point means volume integration, a circle means integration in the space of directions and a rectangle a linear integration along a given direction. Top: variables and integrations in (70). Bottom: variables and integrations used in going from (74) to (75).

Introducing (66) and (67) (with $\mathbf{r}_{a}=\mathbf{r}_{s}=\mathbf{r}_{s}$ for $\left|\psi^{s}\right|^{2}$ ) in (59) we obtain

$$
\left\langle\psi^{a} \psi^{b *}\right\rangle=\left\langle\psi^{a}\right\rangle\left\langle\psi^{b *}\right\rangle+\Gamma_{a b}
$$

with 


$$
\Gamma_{a b}=\rho \int d^{3} \mathbf{r}_{s} \frac{e^{i K\left|\mathbf{r}_{a}-\mathbf{r}_{s}\right|-i K^{*}\left|\mathbf{r}_{b}-\mathbf{r}_{s}\right|}}{\left|\mathbf{r}_{a}-\mathbf{r}_{s}\right|\left|\mathbf{r}_{b}-\mathbf{r}_{s}\right|} \int d \hat{\mathbf{s}}_{s} f\left(\hat{\mathbf{s}}_{s-a}, \hat{\mathbf{s}}_{s}\right) f^{*}\left(\hat{\mathbf{s}}_{s-b}, \hat{\mathbf{s}}_{s}\right) I\left(\mathbf{r}_{s}, \hat{\mathbf{s}}_{s}\right) .
$$

The representation of the variables and integrations used in this expression are depicted in Figure 21(top).

The next step is to introduce the centre and difference vectors in the above expression through the approximations

$$
\begin{gathered}
\left|\mathbf{r}_{a}-\mathbf{r}_{s}\right| \approx\left|\mathbf{r}_{c a b}-\mathbf{r}_{s}\right|+\frac{1}{2} \mathbf{r}_{d a b} \cdot \hat{\mathbf{s}}_{s-c a b} \\
\left|\mathbf{r}_{b}-\mathbf{r}_{s}\right| \approx\left|\mathbf{r}_{c a b}-\mathbf{r}_{s}\right|-\frac{1}{2} \mathbf{r}_{d a b} \cdot \hat{\mathbf{s}}_{s-c a b} \\
\frac{1}{\left|\mathbf{r}_{a}-\mathbf{r}_{s}\right|\left|\mathbf{r}_{b}-\mathbf{r}_{s}\right|} \approx \frac{1}{\left|\mathbf{r}_{c a b}-\mathbf{r}_{s}\right|^{2}}
\end{gathered}
$$

Consistently with the previous conventions, the subscript $s-c a b$ means the direction from point $s$ to the centre point between $a$ and $b$. These approximations are valid for $\left|\mathbf{r}_{d a b}\right| \ll\left|\mathbf{r}_{a}-\mathbf{r}_{s}\right|,\left|\mathbf{r}_{b}-\mathbf{r}_{s}\right|$. Since the magnitude of $\left|\mathbf{r}_{d a b}\right|$ is generally limited within the correlation distance of the field, these assumptions are justified in almost all parts of the medium except in a small volume near the observation points $a$ and $b$. It should be noted that these approximations are consistent with taking $\hat{\mathbf{s}}_{s-a}, \hat{\mathbf{s}}_{s-b} \approx \hat{\mathbf{s}}_{s-c a b}$, which will be assumed.

Using the assumptions (71) to (73), one obtains

$$
\Gamma_{a b}=\rho \int d^{3} \mathbf{r}_{s} \frac{e^{i K_{r} \hat{\mathbf{s}}_{s-c a b} \cdot \mathbf{r}_{d a b}-\rho \sigma t\left|\mathbf{r}_{c a b}-\mathbf{r}_{s}\right|}}{\left|\mathbf{r}_{c a b}-\mathbf{r}_{s}\right|^{2}} \int d \hat{\mathbf{s}}_{s}\left|f\left(\hat{\mathbf{s}}_{s-c a b}, \hat{\mathbf{s}}_{s}\right)\right|^{2} I\left(\mathbf{r}_{s}, \hat{\mathbf{s}}_{s}\right),
$$

where use have been made of $2 \operatorname{Im} K=(4 \pi \rho / k) \operatorname{Im} f(0)=\rho \sigma_{t}$, where $\sigma_{t}=\sigma_{a}+\sigma_{s}$ is the extinction cross-section and $\sigma_{a}$ the absorption cross-section.

To group (67) and (68) with (74) one must rewrite the latter in order to have an angular integration $d \hat{\mathbf{s}}_{c a b}$. The solution is to make a change of variables. This may be better understood with the aid of Figure 11 (bottom). Until now all the vectors have been referenced to an unspecified system of axes with origin $\mathcal{O}$. If we now change the origin of coordinates to the point $\mathbf{r}_{c a b}$ (the centre point between $a$ and $b$ ), the vector $\mathbf{r}_{s}$ (old coordinates) will be represented by a new vector $\mathbf{s}$ (new coordinates) such that $\mathbf{s}=\mathbf{r}_{s}-\mathbf{r}_{c a b}$. Then $\int d^{3} \mathbf{r}_{s}=\int s^{2} d s \int d \hat{\mathbf{s}}_{c a b}, \hat{\mathbf{s}}_{s-c a b} \rightarrow \hat{\mathbf{s}}_{c a b}$ and $\exp \left(i K_{r} \hat{\mathbf{s}}_{s-c a b} \cdot \mathbf{r}_{d a b}\right) \rightarrow \exp \left(i K_{r} \hat{\mathbf{s}}_{c a b} \cdot \mathbf{r}_{d a b}\right)$. This allows to write (67), (68) and (74) with the same angular integration in $d \hat{\mathbf{s}}_{c a b}$. Equation (69) then allows to factorization of $\int \exp \left(i K_{r} \hat{\mathbf{s}}_{c a b} \cdot \mathbf{r}_{d a b}\right)(\cdots) d \hat{\mathbf{s}}_{c a b}$ and becomes 


$$
I\left(\mathbf{r}_{c a b}, \hat{\mathbf{s}}_{c a b}\right)=I_{c}\left(\mathbf{r}_{c a b}, \hat{\mathbf{s}}_{c a b}\right)+\rho \int_{\mathbf{r}_{0}}^{\mathbf{r}_{c a b}} d s e^{-\rho \sigma_{t} s} \int d \hat{\mathbf{s}}_{s}\left|f\left(\hat{\mathbf{s}}_{c a b}, \hat{\mathbf{s}}_{s}\right)\right|^{2} I\left(\mathbf{s}+\mathbf{r}_{c a b}, \hat{\mathbf{s}}_{s}\right) .
$$

Since we have factored out the $d \hat{\mathbf{s}}_{c a b}$ integral, the above expression is valid for each direction $\hat{\mathbf{s}}_{c a b}$. The integration in $s$ is done for all points $\mathbf{r}_{s}$ lying along a line in that direction, whose extremes are $\mathbf{r}_{c a b}$ (the new origin) and a point in the border of $V$, along this line, represented by $\mathbf{r}_{0}$. At this point we may drop the subscript $c a b$ and apply the directional derivative $(d / d s)=\hat{\mathbf{s}} \cdot \nabla \mathbf{r}$. The coherent intensity is expected to obey

$$
\frac{d}{d s} I_{c}(\mathbf{r}, \hat{\mathbf{s}})=-\rho \sigma_{t} I_{c}(\mathbf{r}, \hat{\mathbf{s}})
$$

while in the last term we take the integrand at the point $\mathbf{r}_{s}=\mathbf{r}$, which means $\mathbf{s}=\mathbf{r}_{s}-\mathbf{r}=0$ (see Figure 21(bottom): $\mathbf{s}$ is the vector from point $c a b$ to point $s$ ). We finally obtain the equation of transfer:

$$
\frac{d}{d s} I(\mathbf{r}, \hat{\mathbf{s}})=-\rho \sigma_{t} I_{c}(\mathbf{r}, \hat{\mathbf{s}})+\rho \sigma_{s} \int d \hat{\mathbf{s}}^{\prime} p\left(\hat{\mathbf{s}}, \hat{\mathbf{s}}^{\prime}\right) I\left(\mathbf{r}, \hat{\mathbf{s}}^{\prime}\right)
$$

where we have introduced the phase function given by $p\left(\hat{\mathbf{s}}, \hat{\mathbf{s}}^{\prime}\right)=\left|f\left(\hat{\mathbf{s}}, \hat{\mathbf{s}}^{\prime}\right)\right|^{2} / \sigma_{s}$. This is the equation for stationary scatterers, but the equation for the time-varying intensity may be obtained by a similar process [7].

\section{A.3. Derivation of the transport equation for correlated scatterers}

We now include the last term of (63).We call it $\Gamma_{a b}^{(2)}$ The same conventions are applied and we use again the far-field approximation (66) and the relation (67). The geometry for the calculations presented in this section are depicted in Figure 22. Also, we assume that $G\left(\mathbf{r}_{s}, \mathbf{r}_{t}\right)=G\left(\mathbf{r}_{s}-\mathbf{r}_{t}\right)=G\left(\mathbf{r}_{d s t}\right)$. We obtain

$$
\begin{gathered}
\Gamma_{a b}^{(2)}=-\rho^{2} \int d^{3} \mathbf{r}_{s} d^{3} \mathbf{r}_{t} \frac{e^{i K\left|\mathbf{r}_{a}-\mathbf{r}_{s}\right|-i K^{*}\left|\mathbf{r}_{b}-\mathbf{r}_{s}\right|}}{\left|\mathbf{r}_{a}-\mathbf{r}_{s}\right|\left|\mathbf{r}_{b}-\mathbf{r}_{s}\right|} \\
\int d \hat{\mathbf{s}}_{c s t} f\left(\hat{\mathbf{s}}_{s-a}, \hat{\mathbf{s}}_{s}\right) f^{*}\left(\hat{\mathbf{s}}_{t-b}, \hat{\mathbf{s}}_{t}\right) I\left(\mathbf{r}_{c s t}, \hat{\mathbf{s}}_{c s t}\right) G\left(\mathbf{r}_{d s t}\right) e^{i K_{r} \hat{\mathbf{s}}_{c s t} \cdot \mathbf{r}_{d s t}},
\end{gathered}
$$

where $\mathbf{r}_{c s t}=\left(\mathbf{r}_{s}+\mathbf{r}_{t}\right) / 2, \mathbf{r}_{d s t}=\mathbf{r}_{s}-\mathbf{r}_{t}$ and $\hat{\mathbf{s}}_{c s t}$ represents a generic direction centred on point $\mathbf{r}_{c s t}$. The geometry implicit is depicted in Figure 22(a), top. 

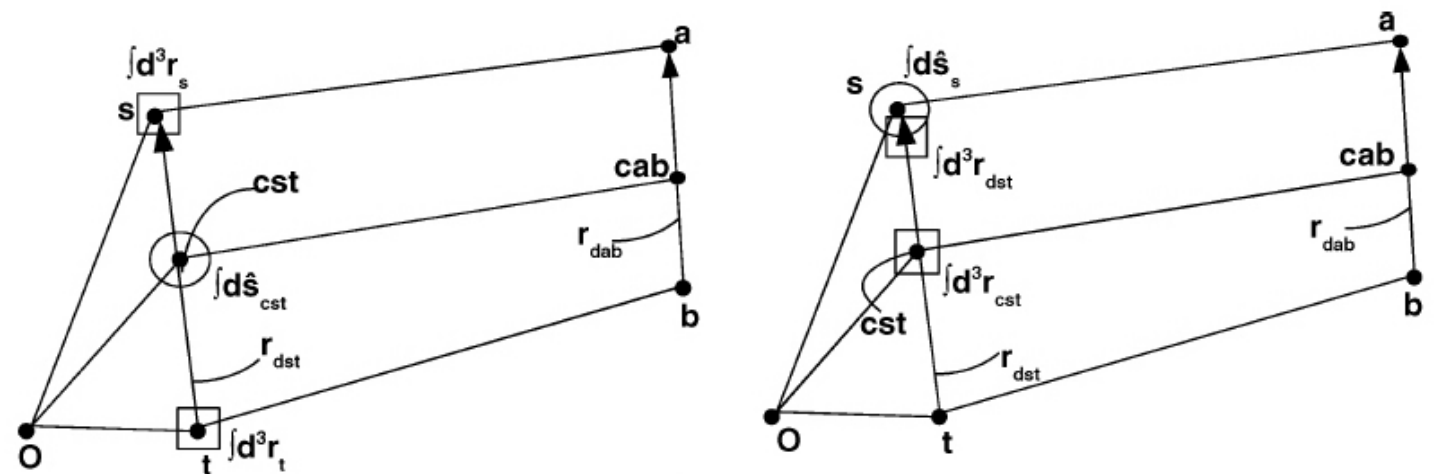

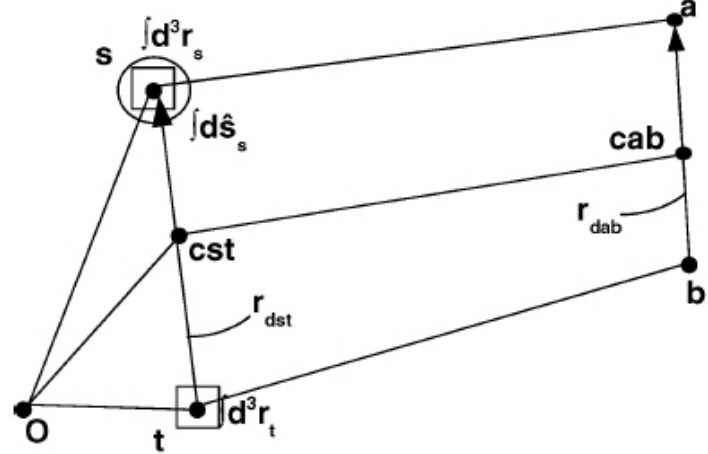

(a)

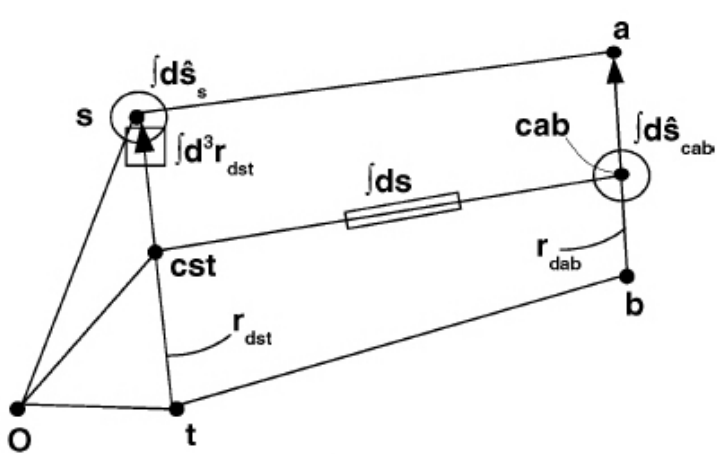

(b)

Figure 22: Geometry used in Section A.3. A square around a given point means volume integration, a circle means integration in the space of directions and a rectangle a linear integration along a given direction. (a) Top: variables and integrations in (78). (a) Bottom: variables and integrations in (79). (b) Top: change of integration variables, from $\mathbf{r}_{s}, \mathbf{r}_{t}$ to $\mathbf{r}_{c s t}, \mathbf{r}_{d s t}$. (b) Bottom: variables and integrations used to obtain (85).

Finally, we also assume that the group of source points ( $s$ and $t$ ) and the group of observation points $(a$ and $b$ ) are separated in such a way that the distance between points of the same group $\left(\left|\mathbf{r}_{s}-\mathbf{r}_{t}\right|\right.$ and $\left.\left|\mathbf{r}_{a}-\mathbf{r}_{b}\right|\right)$ is much smaller than the distance between the groups (well represented by $\left|\mathbf{r}_{c s t}-\mathbf{r}_{c a b}\right|$ ). This is the natural extension of the assumptions that led to (71)-(73). In this case it is legitimate to approximate $\hat{\mathbf{s}}_{t} \approx \hat{\mathbf{s}}_{s}, \hat{\mathbf{s}}_{s-a}, \hat{\mathbf{s}}_{t-b} \approx \hat{\mathbf{s}}_{c s t-c a b}$ and $\hat{\mathbf{s}}_{c s t} \approx \hat{\mathbf{s}}_{s}$, which gives

$$
\begin{gathered}
\Gamma_{a b}^{(2)}=-\rho^{2} \int d^{3} \mathbf{r}_{s} d^{3} \mathbf{r}_{t} \frac{e^{i K\left|\mathbf{r}_{a}-\mathbf{r}_{s}\right|-i K^{*}\left|\mathbf{r}_{b}-\mathbf{r}_{s}\right|}}{\left|\mathbf{r}_{a}-\mathbf{r}_{s}\right|\left|\mathbf{r}_{b}-\mathbf{r}_{s}\right|} \\
\int d \hat{\mathbf{s}}_{s}\left|f\left(\hat{\mathbf{s}}_{c s t-c a b}, \hat{\mathbf{s}}_{s}\right)\right|^{2} I\left(\mathbf{r}_{c s t}, \hat{\mathbf{s}}_{s}\right) G\left(\mathbf{r}_{d s t}\right) e^{i K_{r} \hat{\mathbf{s}}_{s} \cdot \mathbf{r}_{d s t}}
\end{gathered}
$$


The geometry implicit for this expression is represented in Figure 22(a), bottom.

The approximations corresponding to (71)-(73) are now

$$
\begin{gathered}
\left|\mathbf{r}_{a}-\mathbf{r}_{s}\right| \approx\left|\mathbf{r}_{c a b}-\mathbf{r}_{c s t}\right|+\frac{1}{2} \hat{\mathbf{s}}_{c s t-c a b} \cdot\left(\mathbf{r}_{d a b}-\mathbf{r}_{d s t}\right) \\
\left|\mathbf{r}_{b}-\mathbf{r}_{t}\right| \approx\left|\mathbf{r}_{c a b}-\mathbf{r}_{c s t}\right|-\frac{1}{2} \hat{\mathbf{s}}_{c s t-c a b} \cdot\left(\mathbf{r}_{d a b}-\mathbf{r}_{d s t}\right) \\
\frac{1}{\left|\mathbf{r}_{a}-\mathbf{r}_{s}\right|\left|\mathbf{r}_{b}-\mathbf{r}_{t}\right|} \approx \frac{1}{\left|\mathbf{r}_{c a b}-\mathbf{r}_{c s t}\right|^{2}}
\end{gathered}
$$

leading to

$$
\begin{gathered}
\Gamma_{a b}^{(2)}=-\rho^{2} \int d^{3} \mathbf{r}_{s} d^{3} \mathbf{r}_{t} \frac{e^{i K_{r} \hat{\mathbf{s}}_{c s t-c a b} \cdot\left(\mathbf{r}_{d a b}-\mathbf{r}_{d s t}\right)-\rho \sigma_{t}\left|\mathbf{r}_{c a b}-\mathbf{r}_{c s t}\right|}}{\left|\mathbf{r}_{c a b}-\mathbf{r}_{c s t}\right|^{2}} \\
\cdot \int d \hat{\mathbf{s}}_{s}\left|f\left(\hat{\mathbf{s}}_{c s t-c a b}, \hat{\mathbf{s}}_{s}\right)\right|^{2} I\left(\mathbf{r}_{c s t}, \hat{\mathbf{s}}_{s}\right) G\left(\mathbf{r}_{d s t}\right) e^{i K_{r} \hat{\mathbf{s}}_{s} \cdot \mathbf{r}_{d s t}}
\end{gathered}
$$

We change the spatial variables of integration from $\mathbf{r}_{s}, \mathbf{r}_{t}$ to $\mathbf{r}_{c s t}, \mathbf{r}_{d s t}$ (Jacobian $=1$; see Figure 22(b), top) and shift the origin of the coordinates from $\mathcal{O}$ to point $\mathbf{r}_{c a b}$. Then we define the new variable $\mathbf{s}=\mathbf{r}_{c s t}-\mathbf{r}_{c a b}$, implying $\int d \mathbf{r}_{c s t}=\int s^{2} d s \int d \hat{\mathbf{s}}_{c a b}$, $\hat{\mathbf{s}}_{c s t-c a b} \rightarrow \hat{\mathbf{s}}_{c a b}$ and $\exp \left(i K_{r} \hat{\mathbf{s}}_{c s t-c a b} \cdot \mathbf{r}_{d a b}\right) \rightarrow \exp \left(i K_{r} \hat{\mathbf{s}}_{c a b} \cdot \mathbf{r}_{d a b}\right)$. This process may be better understood with the aid of Figure 22(b), bottom. When this new term is added to (69),

$$
\left\langle\psi^{a} \psi^{b *}\right\rangle=\left\langle\psi^{a}\right\rangle\left\langle\psi^{b *}\right\rangle+\Gamma_{a b}+\Gamma_{a b}^{(2)},
$$

it is possible again to factorize $\int \exp \left(i K_{r} \hat{\mathbf{s}}_{c a b} \cdot \mathbf{r}_{d a b}\right)(\cdots) d \hat{\mathbf{s}}_{c a b}$, leading to a new form of (75) with the extra term

$$
\begin{gathered}
-\rho^{2} \int_{\mathbf{r}_{0}}^{\mathbf{r}_{c a b}} d s d^{3} \mathbf{r}_{d s t} e^{-\rho \sigma_{t} s} \\
\cdot \int d \hat{\mathbf{s}}_{s}\left|f\left(\hat{\mathbf{s}}_{c a b}, \hat{\mathbf{s}}_{s}\right)\right|^{2} G\left(\mathbf{r}_{d s t}\right) I\left(\mathbf{s}+\mathbf{r}_{c a b}, \hat{\mathbf{s}}_{s}\right) e^{i K_{r}\left(\hat{\mathbf{s}}_{c a b}-\hat{\mathbf{s}}_{s}\right) \cdot \mathbf{r}_{d s t}}
\end{gathered}
$$

It is now easy to associate this extra term with the last term of (75), which becomes (dropping the subscript $c a b$ and changing again the name $\hat{\mathbf{s}}_{s} \rightarrow \hat{\mathbf{s}}^{\prime}$ )

$$
I(\mathbf{r}, \hat{\mathbf{s}})=I_{c}(\mathbf{r}, \hat{\mathbf{s}})+\rho \int_{\mathbf{r}_{0}}^{\mathbf{r}} d s e^{-\rho \sigma_{t} s} \int d \hat{\mathbf{s}}^{\prime}\left|f\left(\hat{\mathbf{s}}, \hat{\mathbf{s}}^{\prime}\right)\right|^{2}\left[1-\Gamma_{2}\left(\hat{\mathbf{s}}, \hat{\mathbf{s}}^{\prime}\right)\right] I\left(\mathbf{s}+\mathbf{r}, \hat{\mathbf{s}}^{\prime}\right) .
$$


with

$$
\Gamma_{2}\left(\hat{\mathbf{s}}, \hat{\mathbf{s}}^{\prime}\right)=\frac{\rho}{\left|f\left(\hat{\mathbf{s}}, \hat{\mathbf{s}}^{\prime}\right)\right|^{2}} \int d^{3} \mathbf{R} e^{i K_{r}\left(\hat{\mathbf{s}}-\hat{\mathbf{s}}^{\prime}\right) \cdot \mathbf{R}} G(\mathbf{R}) .
$$

The steps from (75) to (77) are applicable exactly in the same way, as long as we substitute $p\left(\hat{\mathbf{s}}, \hat{\mathbf{s}}^{\prime}\right)$ by

$$
P\left(\hat{\mathbf{s}}, \hat{\mathbf{s}}^{\prime}\right)=p\left(\hat{\mathbf{s}}, \hat{\mathbf{s}}^{\prime}\right)\left[1-\Gamma_{2}\left(\hat{\mathbf{s}}, \hat{\mathbf{s}}^{\prime}\right)\right]
$$

Hence, the equation of transfer for correlated scatterers is, within the approximations presented,

$$
\frac{d}{d s} I(\mathbf{r}, \hat{\mathbf{s}})=-\rho \sigma_{t} I_{c}(\mathbf{r}, \hat{\mathbf{s}})+\rho \sigma_{s} \int d \hat{\mathbf{s}}^{\prime} P\left(\hat{\mathbf{s}}, \hat{\mathbf{s}}^{\prime}\right) I\left(\mathbf{r}, \hat{\mathbf{s}}^{\prime}\right)
$$

The equation is formally the same as in the case on uncorrelated scatterers. However, $P\left(\hat{\mathbf{s}}, \hat{\mathbf{s}}^{\prime}\right)$ is not the phase function but a effective phase function. This means that the formalism of the transport equation, especially the diffusion approximation, may be applied. A brief discussion of this feature and the implications for this work were already presented in the second to fourth paragraphs of the Introduction, and we refer the reader to those lines. 